Supplement of Atmos. Chem. Phys., 21, 16293-16317, 2021

https://doi.org/10.5194/acp-21-16293-2021-supplement

(C) Author(s) 2021. CC BY 4.0 License.

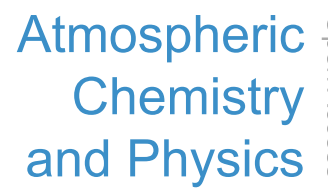

(c) (i)

and Physics

Supplement of

\title{
Nighttime and daytime dark oxidation chemistry in wildfire plumes: an observation and model analysis of FIREX-AQ aircraft data
}

Zachary C. J. Decker et al.

Correspondence to: Steven S. Brown (steven.s.brown@noaa.gov)

The copyright of individual parts of the supplement might differ from the article licence. 


\section{Supporting Text}

\section{UW I- HR ToF CIMS Mass Assignments of $\mathrm{C}_{7} \mathrm{H}_{8} \mathrm{O}_{2}$}

1245 We see significant signal at the mass corresponding to $\mathrm{C}_{7} \mathrm{H}_{8} \mathrm{O}_{2}$. There are at least two explanations for this mass: methylcatechol or guaiacol or any mixture in-between. The time series of $\mathrm{C}_{7} \mathrm{H}_{8} \mathrm{O}_{2}$ suggests it is a primary emission (SI Figure 5 and SI Figure 6). Current BB emissions literature does not list methylcatechol as a detected gas-phase emission (Hatch et al., 2015; Koss et al., 2018), however emissions collected on Teflon filters with subsequent GCxGC analysis show evidence for methylcatechol (Hatch et al., 2018). Both guaiacol and methylcatechol are highly reactive to $\mathrm{NO}_{3}, \mathrm{OH}$, and $\mathrm{O}_{3}$. Therefore,

1250 accurately determining its identity and thus mixing ratio, is important to both constraining the model and comparing it to observations. Collection of smoke during the Castle and Cow plume by a Tenax cartridge sampler with subsequent GCxGC analysis shows no evidence for methylcatechol or guaiacol above detection limits in the Castle plume, but some evidence for guaiacol at roughly $\sim 0.06 \mathrm{ppbv}$ in the Cow plume. Using calibrations for the UW I- $\mathrm{HR}$ ToF CIMS $\mathrm{C}_{7} \mathrm{H}_{8} \mathrm{O}_{2}$ signal for guaiacol and methylcatechol (described in the SI of Palm et al., 2020), we determined that methycatechol is the most likely assignment despite its absence by the Tenax cartridge sampler. If the mass was entirely due to guaiacol, then $\sim 0.06$ ppbv would appear as $<1$ normalized count per second on the UW I ${ }^{-}$HR ToF CIMS, while we observe 1,000 - 10,000 normalized counts per second. In other words, iodide is very weakly sensitive to guaiacol and we do not expect to detect a mixing ratio of $0.06 \mathrm{ppbv}$. Assigning $\mathrm{C}_{7} \mathrm{H}_{8} \mathrm{O}_{2}$ to methylcatechol corresponds to observations of $0.1-1$ ppbv (SI Figure 5 and SI Figure 6) and an emission ratio of 0.4 ppbv ppmv ${ }^{-1} \mathrm{CO}$, or $1 / 3^{\text {rd }}$ that of the catechol emission ratio. This is consistent with the same assignment of $\mathrm{C}_{7} \mathrm{H}_{8} \mathrm{O}_{2}$ and emission ratios observed in (Palm et al., 2020). Lastly, while we expect formation of methylcatechol from cresol oxidation by $\mathrm{OH}$, our box model shows this formation pathway is negligible.

\section{Expansion of Phenolic Mechanisms Description}

Phenol and cresol oxidation have been studied in greater detail than their oxidation products such as catechols and methylcatechols (Calvert et al., 2011). Thus, in order to update the phenolic chemistry, where literature values are unavailable we extrapolate phenol and cresol branching ratios, rate coefficients, and products for catechol, methylcatechol, and the three isomers of dimethylcatechol. As an example, SI Figure 14 shows our reaction mechanisms involving catechol. Reactions in black represent reactions already in the MCM, while reactions in brown represent added reactions.

The reaction of phenol and cresol $+\mathrm{OH}$ is known to form catechol and methylcatechol, respectively, by $\mathrm{OH}$ addition to the ring (Olariu et al., 2002). Similarly, the formation of trihydroxybenzene from catechol was suggested by Nakao et al., 2011 and trihydroxy toluene was identified as an oxidation product from methylcatechol by Schwantes et al., 2017. Further, reactions of phenol and cresol with $\mathrm{OH}$ are also known to produce benzoquinones (Olariu et al., 2002) and Schwantes et al., 2017 identified hydroxymethyl benzoquinone from methylcatechol. Finewax et al., 2018 report the yield of nitrocatechol from $\mathrm{OH}$ 
oxidation of catechol to be $30 \pm 3 \%$. Despite these recent studies, $\mathrm{MCM}$ v3.3.1 assumes, under high $\mathrm{NO}_{2}$ conditions, a 100\% yield of nitrocatechol and nitromethylcatechol from $\mathrm{OH}$-initiated oxidation of catechol and methylcatechol, respectively.

1275 In the updated phenolic mechanism used here, the rate coefficients and branching ratios are taken from the literature when possible, but estimated from analogous reactions when unavailable. $\mathrm{OH}$-initiated oxidation of catechol, methylcatechol, and dimethylcatechols is assumed to form $30 \%$ nitrocatechol, nitromethylcatechol, and nitrodimethylcatechol, respectively, under high $\mathrm{NO}_{2}$ conditions as extrapolated from Finewax et al., 2018. The remaining 70\% of products from $\mathrm{OH}$-initiated catechol, methylcatechol, and dimethylcatechols produce trihydroxybenzene and hydroxybenzoquinone type products consistent with recent work for catechol and methyl catechol oxidation (Nakao et al., 2011 and Schwantes et al., 2017). To calculate the branching ratio between hydroxybenzoquinone and trihydroxybenzene from catechol $+\mathrm{OH}$, we scale the yields found for each type from literature for phenol $+\mathrm{OH}$ (Nakao et al., 2011; Olariu et al., 2002). The result is an estimated 3\% yield of hydroxybenzoquinone and a $67 \%$ yield of trihydroxybenzene. Similarly, for methylcatechol and dimethylcatechol $+\mathrm{OH}$, we scale the yields from literature for each type from $o$-cresol + OH (Nakao et al., 2011; Olariu et al., 2002).

1285 Finewax et al., 2018 report the yield of nitrocatechol from $\mathrm{NO}_{3}$ oxidation of catechol to be $91 \pm 6 \%$. Olariu et al. also found benzoquinone formation from $\mathrm{NO}_{3}+$ cresol (Olariu et al., 2013). The mechanism by which benzoquinones are formed by $\mathrm{NO}_{3}$ oxidation of catechol is uncertain. Olariu et al. state that unpublished work finds no evidence for benzoquinone products from phenol $+\mathrm{NO}_{3}$, while Bolzacchini et al. find evidence for benzoquinone production from $\mathrm{NO}_{3}+$ phenol if $\mathrm{O}_{3}$ is present (Bolzacchini et al., 2001). We assume catechol, methylcatechol, and dimethylcatechols $+\mathrm{NO}_{3}$ forms $91 \%$ nitrocatechol type 1290 product and the remaining 9\% a benzoquinone type product, as seen in SI Figure 14. For simplification in our box model, we group benzoquinone products.

Referring to SI Figure 14, $\mathrm{OH}$ and $\mathrm{NO}_{3}$ reactions with catechol form a catechol radical (CATO), which can then react with either $\mathrm{NO}_{2}$ or $\mathrm{O}_{3}$. Reactions with $\mathrm{NO}_{2}$ form nitrocatechol (NCATECHOL), while reactions with $\mathrm{O}_{3}$ form a catechol-peroxy radical $\left(\mathrm{CATO}_{2}\right)$, which can subsequently react with $\mathrm{HO}_{2}$ to form a catechol-hydroperoxide (CATOOH). The formation of

1295 CATOOH is reversible but our model runs find the lifetime of CATOOH is $>100$ hours meaning CATOOH acts as a permanent loss of $\mathrm{CATO}_{2}$. While our catechol mechanism does not include heterogeneous reactions, it is likely that CATOOH will be lost through aerosol. We find that CATOOH is responsible for $17-26 \%$ of net CATO loss. In other words, $17-26 \%$ of potential nitrocatechol formation is lost to $\mathrm{CATOOH}$. In all model runs, the fraction of potential nitrocatechol lost by $\mathrm{O}_{3}$ increases as the plume ages.

1300 For updates to both $\mathrm{OH}$ - and $\mathrm{NO}_{3}$ - phenolic compound oxidation, we assume similar assumptions for later-generation products and end the oxidation at highly-functionalized products, which will likely form secondary organic aerosol. Below, we provide the mechanism in the form of FACSIMILE, which is a standard output format (.fac) from the MCM and is readable in F0AM v4.0. 


\section{Mechanism}

1305 Note: reactions in red are already included in the MCM with a different yield. When using the below mechanism in conjunction with the MCM, the red reactions will need to be removed from the MCM to avoid duplicate reactions.

\section{Catechols}

$\%$ 1.0D-10*0.3 : CATECHOL $+\mathrm{OH}=\mathrm{CATEC} 1 \mathrm{O}$;

$\%$ 1.0D-10*0.67 : CATECHOL $+\mathrm{OH}=\mathrm{H} 3 \mathrm{BENZENE}+\mathrm{HO} 2$;

$1310 \%$ 1.0D-10*0.03: CATECHOL $+\mathrm{OH}=\mathrm{HPBZQONE}+\mathrm{HO} 2$

$\%$ 3.00D-13 : HPBZQONE + NO3 = NBZQO2 ;

$\%$ 4.6D-12: HPBZQONE + OH = PBZQO2 ;

$\%$ 1.0D-10*0.3 : H3BENZENE + OH = H3BENZENE1O ;

$\%$ 1.0D-10*0.67 : H3BENZENE + OH = H4BENZENE + $\mathrm{HO} 2$;

$1315 \%$ 1.0D-10*0.03 : H3BENZENE $+\mathrm{OH}=\mathrm{H} 2 \mathrm{PBZQONE}+\mathrm{HO}$;

$\%$ 3.00D-13 : H2PBZQONE + NO3 = NBZQO2 ;

$\%$ 4.6D-12 : H2PBZQONE + OH = PBZQO2 ;

$\%$ 2.08D-12 : H3BENZENE1O + NO2 = NH3BENZENE ;

$\%$ 2.86D-13 : H3BENZENE1O + O3 = H3BENZENE1O2 ;

$1320 \% \mathrm{KRO} 2 \mathrm{HO} 2 * 0.770: \mathrm{H} 3 \mathrm{BENZENE} 1 \mathrm{O} 2+\mathrm{HO} 2=\mathrm{HCATEC} 1 \mathrm{OOH}$;

$\%$ KRO2NO : H3BENZENE1O2 + NO = H3BENZENE1O + NO2 ;

$\%$ KRO2NO3 : H3BENZENE1O2 + NO3 = H3BENZENE1O + NO2 ;

$\%$ 8.80D-13*RO2 : H3BENZENE1O2 = H3BENZENE1O ;

$\%$ 9.9D-11*0.91: CATECHOL + NO3 = CATEC1O + HNO3 ;

$\%$ 9.9D-11*0.09: CATECHOL + NO3 = HPBZQONE + HO2 + HNO3 ;

$\%$ 9.9D-11*0.91 : H3BENZENE + NO3 = H3BENZENE1O + HNO3 ;

$\%$ 9.9D-11*0.09: H3BENZENE + NO3 = H2PBZQONE + HO2 + HNO3 ;

\section{Methylcatechols}

$1330 \%$ 2.0D-10*0.3 : MCATECHOL + OH = MCATEC1O ;

$\%$ 2.0D-10*0.64 : MCATECHOL + OH = H3TOLUENE + HO2;

$\%$ 2.0D-10*0.06: MCATECHOL + OH = HPTLQONE + HO2;

$\%$ 1.00D-12: HPTLQONE + NO3 = NPTLQO 2 ;

$\%$ 2.3D-11 : HPTLQONE + OH = PTLQO2 ;

$1335 \%$ 2.0D-10*0.3 : H3TOLUENE + OH = H3TOLUENE1O ;

$\%$ 2.0D-10*0.64 : H3TOLUENE + OH = H4TOLUENE + HO2; 
\% 2.0D-10*0.06 : H3TOLUENE + OH = H2PTLQONE + HO2;

$\%$ 1.00D-12: H2PTLQONE + NO3 = NPTLQO2 ;

$\%$ 2.3D-11 : H2PTLQONE + OH = PTLQO2 ;

$1340 \%$ 2.08D-12: H3TOLUENE1O + NO2 = NH3TOLUENE;

$\%$ 2.86D-13 : H3TOLUENE1O + O3 = H3TOLUENE1O2 ;

$\% \mathrm{KRO} 2 \mathrm{HO} 2 * 0.820: \mathrm{H} 3 \mathrm{TOLUENE} 1 \mathrm{O} 2+\mathrm{HO} 2=\mathrm{HMCATEC} 1 \mathrm{OOH}$;

$\%$ KRO2NO : H3TOLUENE1O2 + NO = H3TOLUENE1O + NO2 ;

$\%$ KRO2NO3 : H3TOLUENE1O2 + NO3 = H3TOLUENE1O + NO2 ;

$1345 \%$ 8.80D-13*RO2 : H3TOLUENE1O2 = H3TOLUENE1O ;

$\%$ 1.7D-10*0.91 : MCATECHOL + NO3 = MCATEC1O + HNO3 ;

$\%$ 1.7D-10*0.09: MCATECHOL + NO3 = HPTLQONE + HO2 + HNO3 ;

$\%$ 1.7D-10*0.91 : H3TOLUENE + NO3 = H3TOLUENE1O + HNO3 ;

$\%$ 1.7D-10*0.09: H3TOLUENE + NO3 = H2PTLQONE + HO2 + HNO3 ;

1350 Dimethylcatechols

$\%$ 2.05D-10*0.3 : OXYCATECH + OH = OXCATEC1O ;

$\%$ 2.05D-10*0.64 : OXYCATECH + OH = H3OXYLENE + HO2;

$\%$ 2.05D-10*0.06 : OXYCATECH + OH = HOXYQONE + HO2;

$\%$ 1.00D-12: HOXYQONE + NO3 = NOXYQO 2 ;

$1355 \%$ 2.35D-10 : HOXYQONE + OH = OXYQO2 ;

$\%$ 2.05D-10*0.3 : H3OXYLENE + OH = H3OXYLENE1O ;

$\%$ 2.05D-10*0.64 : H3OXYLENE + OH = H4OXYLENE + HO2;

$\%$ 2.05D-10*0.06 : H3OXYLENE + OH = H2OXYQONE + HO2;

$\%$ 1.00D-12 : H2OXYQONE + NO3 = NOXYQO2 ;

$1360 \%$ 2.35D-10 : H2OXYQONE + OH = OXYQO2 ;

$\%$ 2.08D-12: H3OXYLENE1O + NO2 = NH3OXYLENE ;

$\%$ 2.86D-13 : H3OXYLENE1O + O3 = H3OXYLENE1O2 ;

$\% \mathrm{KRO} 2 \mathrm{HO} 2 * 0.859: \mathrm{H} 3 \mathrm{OXYLENE} 1 \mathrm{O} 2+\mathrm{HO} 2=\mathrm{HOCATEC} 1 \mathrm{OOH}$;

$\%$ KRO2NO : H3OXYLENE1O2 + NO = H3OXYLENE1O + NO2 ;

$1365 \%$ KRO2NO3 : H3OXYLENE1O2 + NO3 = H3OXYLENE1O + NO2 ;

$\%$ 8.80D-13*RO2 : H3OXYLENE1O2 = H3OXYLENE1O ;

$\%$ 2.01D-10*0.91 : OXYCATECH + NO3 = OXCATEC1O + HNO3 ;

$\%$ 2.01D-10*0.09: OXYCATECH + NO3 = HOXYQONE + HO2 + HNO3 ;

$\%$ 2.01D-10*0.91: H3OXYLENE + NO3 = H3OXYLENE1O + HNO3 ;

$\%$ 2.01D-10*0.09: H3OXYLENE + NO3 $=\mathrm{H} 2 \mathrm{OXYQONE}+\mathrm{HO} 2+\mathrm{HNO} 3$; 
\% 2.05D-10*0.3 : PXYCATECH + OH = PXCATEC1O ;

$\%$ 2.05D-10*0.64 : PXYCATECH + OH = H3PXYLENE + HO2;

$\%$ 2.05D-10*0.06 : PXYCATECH + OH = HPXYQONE + HO2;

$1375 \%$ 1.00D-12: HPXYQONE + NO3 = NPXYQO2 ;

$\%$ 2.35D-11 : HPXYQONE + OH = PXYQO2 ;

$\%$ 2.05D-10*0.3 : H3PXYLENE + OH = H3PXYLENE1O ;

$\%$ 2.05D-10*0.64 : H3PXYLENE + OH = H4PXYLENE + HO2;

$\%$ 2.05D-10*0.06 : H3PXYLENE + OH = H2PXYQONE + HO2;

$1380 \%$ 1.00D-12: H2PXYQONE + NO3 = NPXYQO 2 ;

$\%$ 2.35D-11 : H2PXYQONE + OH = PXYQO2 ;

\% 2.08D-12 : H3PXYLENE1O + NO2 = NH3PXYLENE ;

$\%$ 2.86D-13 : H3PXYLENE1O + O3 = H3PXYLENE1O2 ;

$\% \mathrm{KRO} 2 \mathrm{HO} 2 * 0.859: \mathrm{H} 3 \mathrm{PXYLENE} 1 \mathrm{O} 2+\mathrm{HO} 2=\mathrm{HPCATEC} 1 \mathrm{OOH}$;

$\%$ KRO2NO : H3PXYLENE1O2 + NO = H3PXYLENE1O + NO2 ;

$\%$ KRO2NO3 : H3PXYLENE1O2 + NO3 = H3PXYLENE1O + NO2 ;

$\%$ 8.80D-13*RO2 : H3PXYLENE1O2 = H3PXYLENE1O ;

$\%$ 2.01D-10*0.91 : PXYCATECH + NO3 = PXCATEC1O + HNO3 ;

\% 2.01D-10*0.09: PXYCATECH + NO3 = HPXYQONE + HO2 + HNO3 ;

$1390 \%$ 2.01D-10*0.91 : H3PXYLENE + NO3 = H3PXYLENE1O + HNO3 ;

$\%$ 2.01D-10*0.09: H3PXYLENE + NO3 = H2PXYQONE + HO2 + HNO3 ;

\% 2.05D-10*0.3 : MXYCATECH + OH = MXCATEC1O ;

$\%$ 2.05D-10*0.64 : MXYCATECH + OH = H3MXYLENE + HO2;

$1395 \%$ 2.05D-10*0.06: MXYCATECH + OH = HMXYQONE + HO2;

$\%$ 1.00D-12 : HMXYQONE + NO3 = NMXYQO2 ;

$\%$ 2.35D-11 : HMXYQONE + OH = MXYQO2 ;

$\%$ 2.05D-10*0.3 : H3MXYLENE + OH = H3MXYLENE1O ;

\% 2.05D-10*0.64 : H3MXYLENE + OH = H4MXYLENE + HO2;

$1400 \%$ 2.05D-10*0.06: H3MXYLENE + OH = H2MXYQONE + HO2 ;

$\%$ 1.00D-12: H2MXYQONE + NO3 = NMXYQO2 ;

$\%$ 2.35D-11: H2MXYQONE + OH = MXYQO2 ;

\% 2.08D-12: H3MXYLENE1O + NO2 = NH3MXYLENE ;

\% 2.86D-13 : H3MXYLENE1O + O3 = H3MXYLENE1O2 ; 
$1405 \% \mathrm{KRO} 2 \mathrm{HO} 2 * 0.859: \mathrm{H} 3 \mathrm{MXYLENE} 1 \mathrm{O} 2+\mathrm{HO} 2=\mathrm{HMXCTEC} 1 \mathrm{OOH}$;

$\%$ KRO2NO : H3MXYLENE1O2 + NO = H3MXYLENE1O + NO2 ;

$\%$ KRO2NO3 : H3MXYLENE1O2 + NO3 = H3MXYLENE1O + NO2 ;

$\%$ 8.80D-13*RO2 : H3MXYLENE1O2 = H3MXYLENE1O ;

$\%$ 2.01D-10*0.91: MXYCATECH + NO3 = MXCATEC1O + HNO3 ;

$1410 \%$ 2.01D-10*0.09: MXYCATECH + NO3 = HMXYQONE + HO2 + HNO3 ;

$\%$ 2.01D-10*0.91 : H3MXYLENE + NO3 = H3MXYLENE1O + HNO3 ;

\% 2.01D-10*0.09: H3MXYLENE + NO3 = H2MXYQONE + HO2 + HNO3 ; 


\section{Supporting Figures}

\begin{tabular}{|c|c|c|c|c|}
\hline $\begin{array}{l}\text { SI Table 1: List of instruments and } \\
\text { measurements used in this } \\
\text { analysisMeasurements Used }\end{array}$ & Method & Platform & $\begin{array}{l}\text { Sample } \\
\text { Frequency }\end{array}$ & Reference \\
\hline $\begin{array}{l}\mathrm{HONO}, \mathrm{C}_{6} \mathrm{H}_{6} \mathrm{O}, \mathrm{C}_{7} \mathrm{H}_{8} \mathrm{O}, \mathrm{C}_{6} \mathrm{H}_{6} \mathrm{O}_{2} \\
\mathrm{C}_{7} \mathrm{H}_{8} \mathrm{O}_{2}, \mathrm{C}_{6} \mathrm{H}_{5} \mathrm{NO}_{4}, \mathrm{C}_{7} \mathrm{H}_{7} \mathrm{NO}_{4} \\
\mathrm{C}_{6} \mathrm{H}_{5} \mathrm{NO}_{3}, \mathrm{C}_{7} \mathrm{H}_{7} \mathrm{NO}_{3} .\end{array}$ & $\begin{array}{l}\text { University of Washington Iodide High } \\
\text { Resolution Time of Flight Chemical } \\
\text { Ionization Mass Spectrometer (UW I'- } \\
\text { HR ToF CIMS) }\end{array}$ & $\begin{array}{l}\text { Twin } \\
\text { Otter }\end{array}$ & $2 \mathrm{~Hz}$ & (Lee et al., 2014) \\
\hline Guaiacol and methylcatechol. & $\begin{array}{l}\text { Tenax cartridge sampler with subsequent } \\
\text { GCxGC analysis }\end{array}$ & $\begin{array}{l}\text { Twin } \\
\text { Otter }\end{array}$ & $\sim 5 \min$ & $\begin{array}{l}\text { (Hatch et al., 2015; } \\
\text { Mondello et al., } \\
\text { 2008) }\end{array}$ \\
\hline $\mathrm{CO}$ & $\begin{array}{l}\text { Picarro G2401-m, cavity ringdown } \\
\text { spectrometer. }\end{array}$ & $\begin{array}{l}\text { Twin } \\
\text { Otter }\end{array}$ & $0.5 \mathrm{~Hz}$ & (Crosson, 2008) \\
\hline $\mathrm{NO}, \mathrm{NO}_{2}$ and $\mathrm{O}_{3}$. & NCAR chemiluminescence instrument & $\begin{array}{l}\text { Twin } \\
\text { Otter }\end{array}$ & $1 \mathrm{~Hz}$ & (Sparks et al., 2019) \\
\hline Aerosol surface area & $\begin{array}{l}\text { Ultra-high sensitivity aerosol } \\
\text { spectrometer (UHSAS) }\end{array}$ & $\begin{array}{l}\text { Twin } \\
\text { Otter }\end{array}$ & $1 \mathrm{~Hz}$ & (Kupc et al., 2018) \\
\hline $\mathrm{jNO}_{2}$ & $\begin{array}{l}\text { Meteorologie Consult, } \mathrm{GmbH} \text { upward } \\
\text { and downward facing } \mathrm{jNO}_{2} \text { filter } \\
\text { radiometers } \\
\text { Tunable diode laser snectrometer }\end{array}$ & $\begin{array}{l}\text { Twin } \\
\text { Otter } \\
\text { DC-8 }\end{array}$ & $1 \mathrm{~Hz}$ & $\begin{array}{l}\text { (Kupc et al., 2018; } \\
\text { Warneke et al., } \\
\text { 2016) } \\
\text { (Sachse et al. 1991) }\end{array}$ \\
\hline $\mathrm{CO}$ & Cavity enhanced spectrometer & DC-8 & $1 \mathrm{~Hz}$ & (Eilerman et al., \\
\hline $\mathrm{NO}_{2}, \mathrm{NO}_{\mathrm{y}}$, and $\mathrm{O}_{3}$ & NOAA chemiluminescence & DC-8 & $1 \mathrm{~Hz}$ & $\begin{array}{l}\text { 2016) } \\
\text { (Pollack et al., 2010; } \\
\text { Ridley et al., 1992; } \\
\text { Stedman et al., } \\
\text { 1972) }\end{array}$ \\
\hline $\mathrm{NO}_{2}$ & $\begin{array}{l}\text { NOAA broadband Airborne Cavity } \\
\text { Enhanced Spectrometer (ACES) }\end{array}$ & DC-8 & $1 \mathrm{~Hz}$ & (Min et al., 2016) \\
\hline NO & NOAA laser induced fluorescence & DC-8 & $1 \mathrm{~Hz}$ & (Rollins et al., 2020) \\
\hline $\mathrm{HONO}, \mathrm{C}_{6} \mathrm{H}_{6} \mathrm{O}_{2}, \mathrm{C}_{6} \mathrm{H}_{5} \mathrm{NO}_{4}$ & $\begin{array}{l}\text { NOAA Iodide Time of Flight Chemical } \\
\text { Ionization Mass Spectrometer (I- ToF } \\
\text { CIMS) }\end{array}$ & DC-8 & $1 \mathrm{~Hz}$ & $\begin{array}{l}\text { (Neuman et al., } \\
\text { 2016; Veres et al., } \\
\text { 2020) }\end{array}$ \\
\hline $\begin{array}{l}\mathrm{C}_{6} \mathrm{H}_{6} \mathrm{O}, \mathrm{C}_{7} \mathrm{H}_{8} \mathrm{O}, \mathrm{C}_{7} \mathrm{H}_{8} \mathrm{O}_{2}, \mathrm{C}_{7} \mathrm{H}_{8}, \mathrm{C}_{8} \mathrm{H}_{8} \\
\mathrm{C}_{4} \mathrm{H}_{4} \mathrm{O}, \mathrm{C}_{4} \mathrm{H}_{2} \mathrm{O}_{3}, \mathrm{C}_{4} \mathrm{H}_{6} \mathrm{O}, \mathrm{C}_{7} \mathrm{H}_{6} \mathrm{O} \\
\mathrm{C}_{6} \mathrm{H}_{6} .\end{array}$ & $\begin{array}{l}\text { University of Innsbruck Proton Transfer } \\
\text { Reaction Time of Flight Mass } \\
\text { Spectrometer (UIBK PTR ToF MS) }\end{array}$ & DC-8 & $1 \mathrm{~Hz}$ & (Müller et al., 2014) \\
\hline peroxyacetyl nitrate (PAN) & $\begin{array}{l}\text { Thermal dissociation Chemical } \\
\text { Ionization Mass Spectrometer }\end{array}$ & DC-8 & $1 \mathrm{~Hz}$ & (Ro Lee et al., 2020) \\
\hline $\begin{array}{l}\text { Aerosol Size Distribution and Derived } \\
\text { Surface Area }\end{array}$ & $\begin{array}{l}\text { Scanning mobility particle sizer (SMPS) } \\
\text { Laser Aerosol Spectrometer (LAS) }\end{array}$ & DC-8 & $\begin{array}{l}60 \mathrm{sec} \\
1 \mathrm{~Hz}\end{array}$ & $\begin{array}{l}\text { (LAS, n.d.; Moore } \\
\text { et al., 2021; SMPS, } \\
\text { n.d.) }\end{array}$ \\
\hline Photolysis rates listed in SI Table 4 & $\begin{array}{l}\text { Charged-coupled device Actinic Flux } \\
\text { Spectroradiometer (CAFS) }\end{array}$ & DC-8 & $1 \mathrm{~Hz}$ & $\begin{array}{l}\text { (Shetter and Müller, } \\
\text { 1999) }\end{array}$ \\
\hline
\end{tabular}


SI Table 2: List of estimated emission times (UTC) with uncertainty (min) for each plume. Emission times for transects used 1420 to constrain the model are bolded.

Estimated Emission Time at Plume Center (UTC) \pm uncertainty (min)

\begin{tabular}{|c|c|c|c|c|}
\hline Transect & WF 1 (Aug 7 2019) & WF2 & Castle (Aug 22 2019) & Cow (Aug 29 2019) \\
\hline 1 & $23: 01: 04 \pm 5.0 \mathrm{~min}$ & Aug 8 00:36:01 $\pm 8.0 \mathrm{~min}$ & $01: 01: 41 \pm 1.2 \mathrm{~min}$ & 01:30:59 $\pm 71.5 \mathrm{~min}$ \\
\hline 2 & $22: 46: 13 \pm 6.6 \mathrm{~min}$ & Aug 8 00:18:18 $\pm 7.7 \mathrm{~min}$ & $00: 59: 58 \pm 1.4 \mathrm{~min}$ & $01: 27: 45 \pm 68.1 \mathrm{~min}$ \\
\hline 3 & $22: 43: 11 \pm 3.8 \mathrm{~min}$ & Aug 8 00:09:45 $\pm 6.2 \mathrm{~min}$ & $00: 59: 55 \pm 1.7 \mathrm{~min}$ & $01: 30: 34 \pm 55.6 \mathrm{~min}$ \\
\hline 4 & $22: 33: 25 \pm 8.6 \mathrm{~min}$ & Aug 7 23:53:59 $\pm 7.2 \mathrm{~min}$ & $00: 52: 11 \pm 3.8 \mathrm{~min}$ & \\
\hline 5 & $22: 13: 04 \pm 13.7 \mathrm{~min}$ & Aug $723: 29: 05 \pm 12.8 \mathrm{~min}$ & & \\
\hline 6 & $21: 58: 06 \pm 12.8 \mathrm{~min}$ & Aug 7 23:24:59 $\pm 8.2 \mathrm{~min}$ & & \\
\hline 7 & $21: 51: 34 \pm 16.5 \mathrm{~min}$ & Aug $723: 14: 38 \pm 6.2 \mathrm{~min}$ & & \\
\hline 8 & $21: 37: 17 \pm 15.6 \mathrm{~min}$ & Aug $722: 50: 45 \pm 11.4 \mathrm{~min}$ & & \\
\hline 9 & $21: 13: 38 \pm 19.9 \mathrm{~min}$ & Aug $722: 41: 39 \pm 22.5 \mathrm{~min}$ & & \\
\hline 10 & $20: 55: 25 \pm 30.2 \mathrm{~min}$ & & & \\
\hline
\end{tabular}




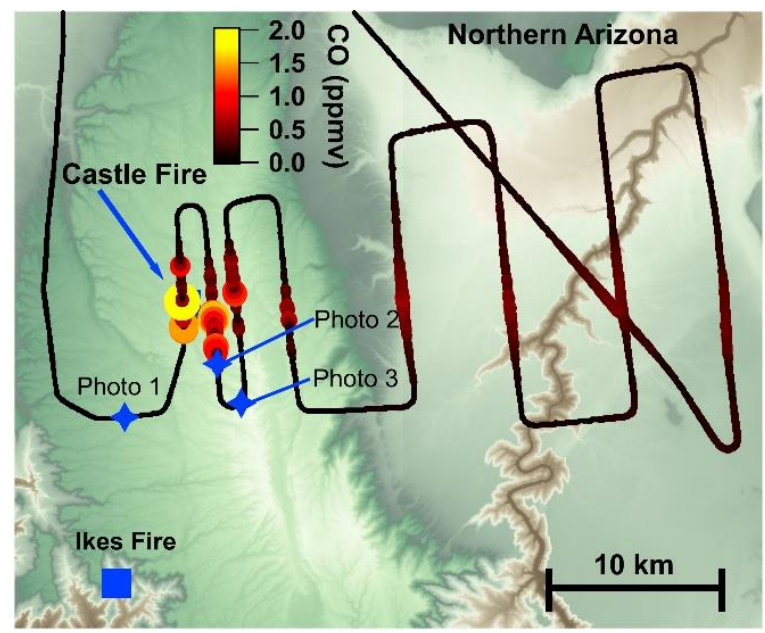

Photo 2

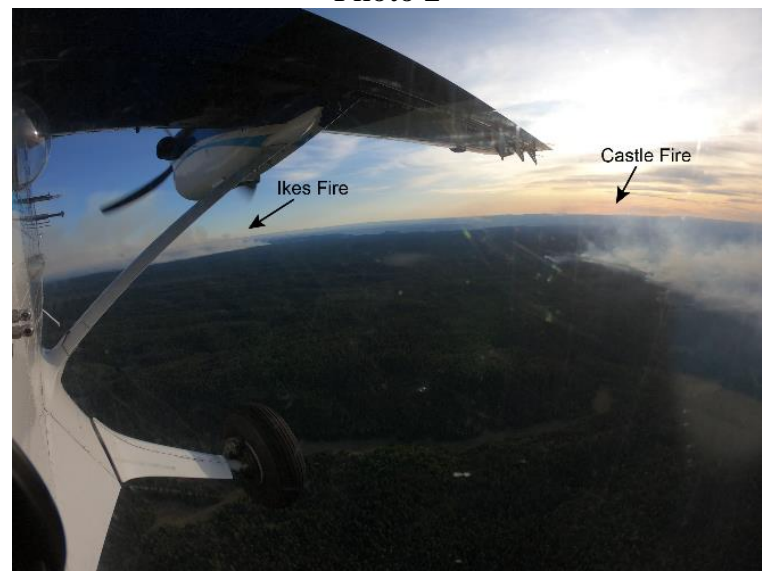

Photo 1

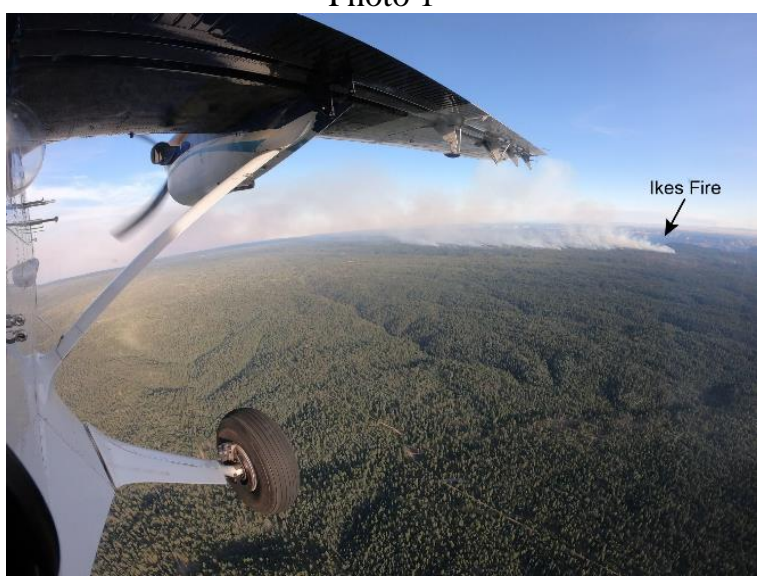

Photo 3

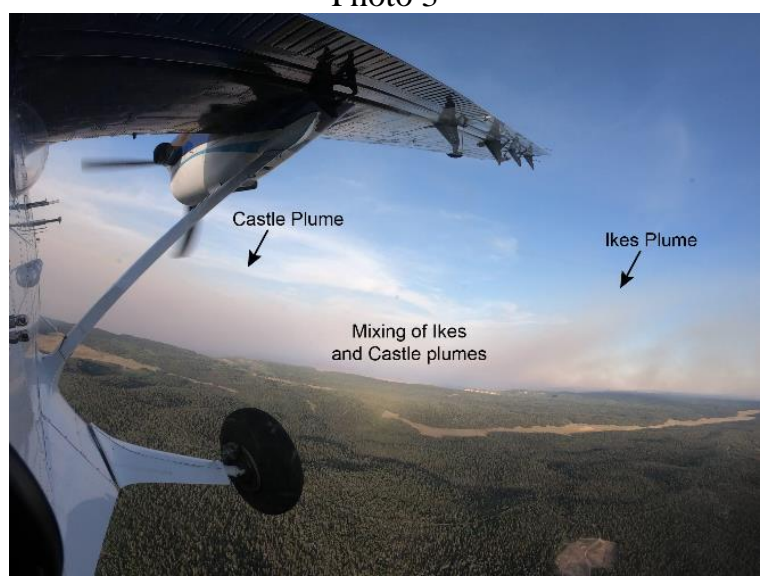

SI Figure 1: Flight map and photos of the Castle plume. Photos indicate the Ikes and Castle fires, which burned near each other and eventually mixed plumes. The first four (of 8) transects of the Castle plume are unmixed with the Ikes plume. 

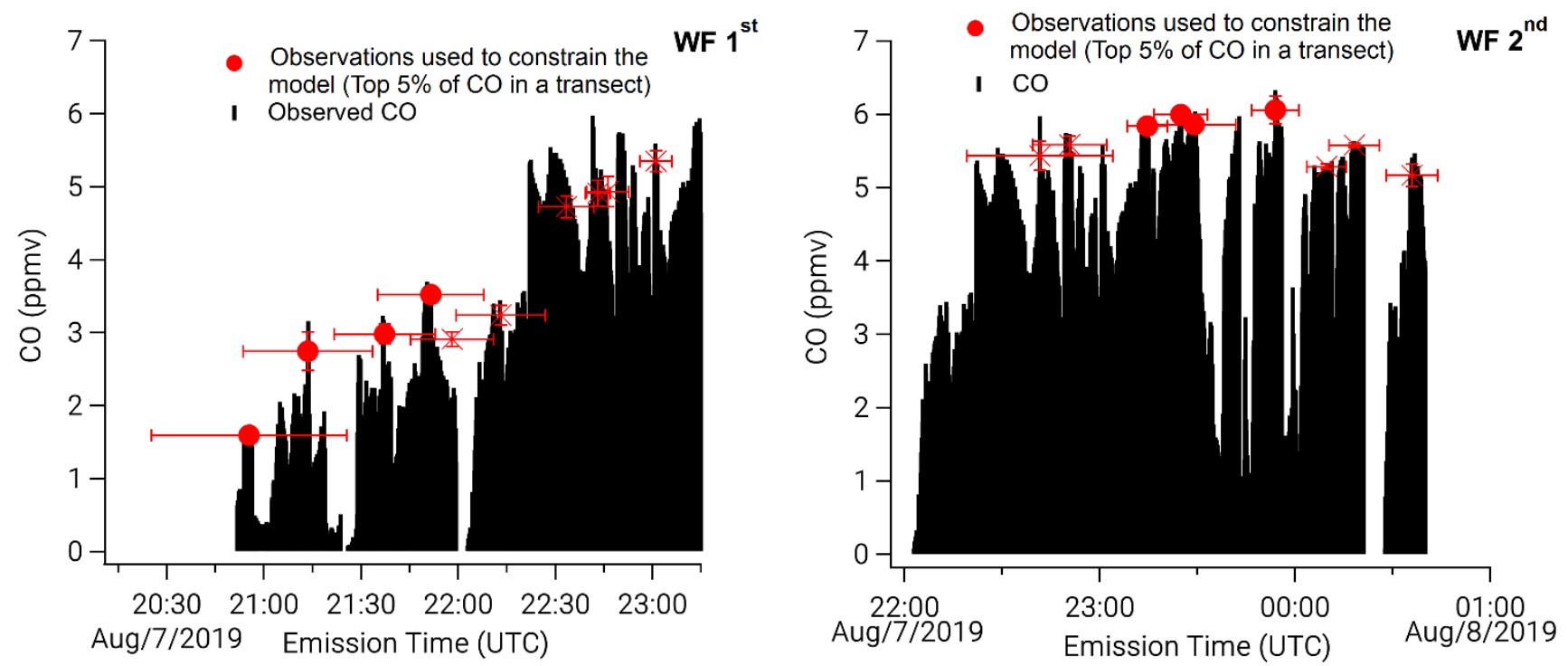

SI Figure 2: Time series of CO (black filled) as a function of emission time. Red markers indicate the top $5 \%$ of CO during a single transect. Filled circles indicate observations chosen to constrain a model run and crosses indicate unused observations. 

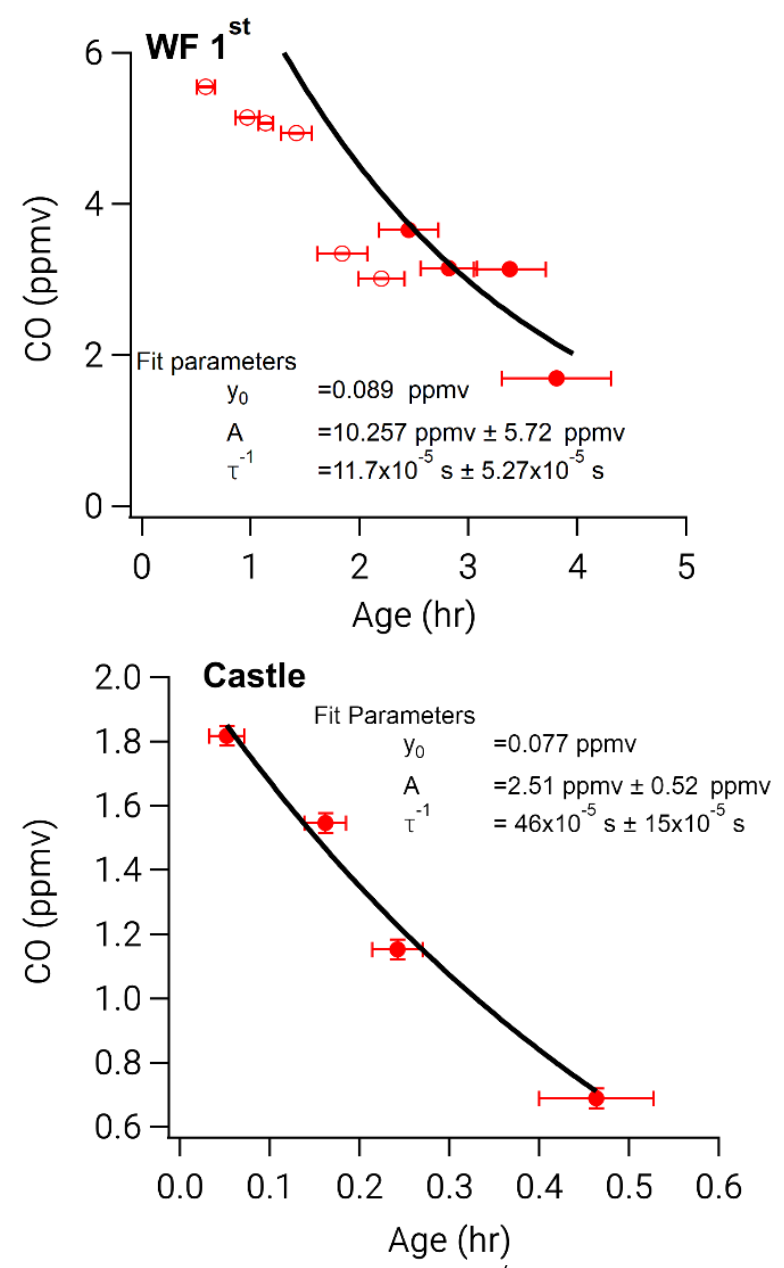
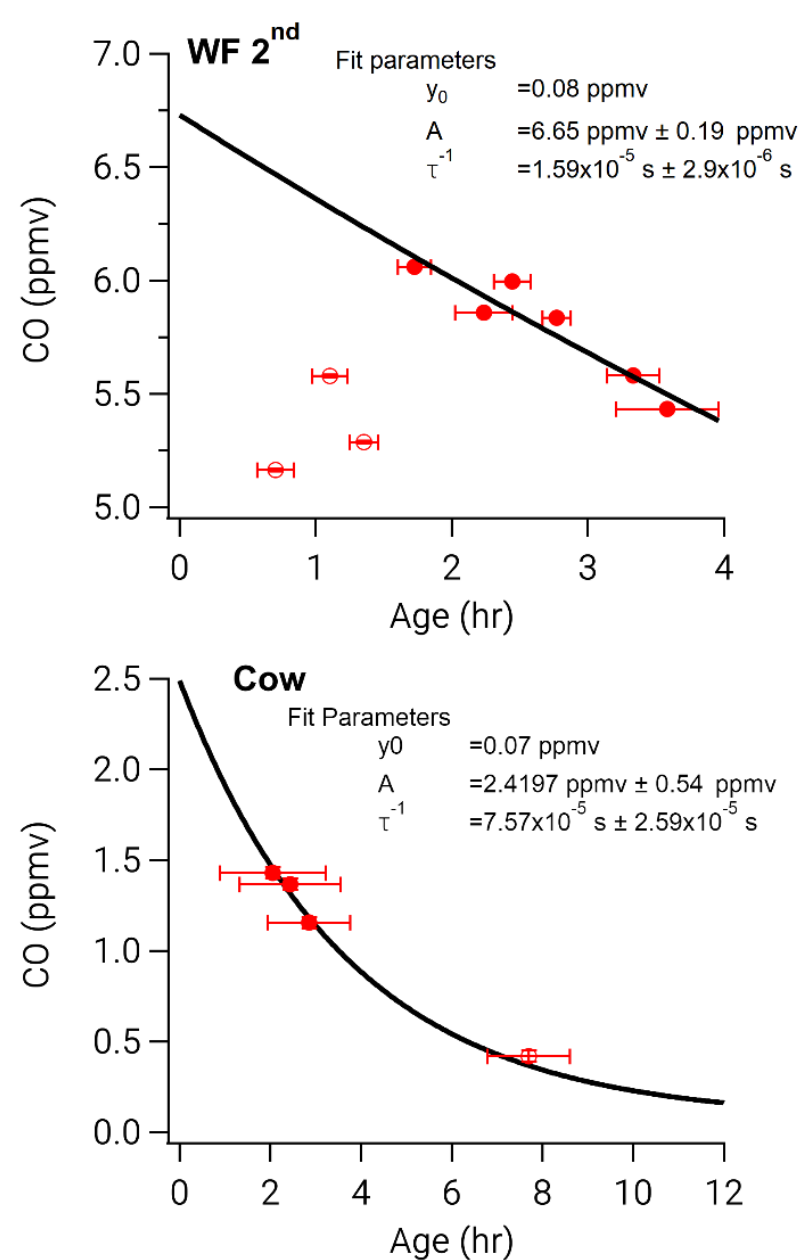

SI Figure 3: Exponential fit $\left(y=A e^{x / \tau}+y_{0}\right)$ to normalized excess mixing ratios (NEMR) of CO used as our best-guess estimate of dilution for each model run. Filled circles indicate observations used to constrain the model run, while open circle indicate observation that are not used. 
SI Table 3: Table of background mixing ratios of $\mathrm{CO}$, NO, $\mathrm{NO}_{2}, \mathrm{HONO}$, and $\mathrm{O}_{3}$ for all model runs. The Dark model run has the same conditions as the WF2 model run.

\begin{tabular}{c|c|c|c|c} 
Compound & WF1 & WF2 & Castle & Cow \\
\hline $\mathrm{CO}(\mathrm{ppmv})$ & 0.089 & 0.080 & 0.124 & 0.070 \\
$\mathrm{NO}(\mathrm{ppbv})$ & 0.0 & 0.0 & 0.0 & 0.0 \\
$\mathrm{NO}_{2}(\mathrm{ppbv})$ & 0.0 & 0.1 & 0.1 & 0.06 \\
$\mathrm{HONO}(\mathrm{ppbv})$ & 0.0 & 0.0 & 0.0 & 0.0 \\
$\mathrm{O}_{3}(\mathrm{ppbv})$ & 96.25 & 84.2 & 90 & 60.7 \\
$\mathrm{k}_{\mathrm{dil}}\left(\times 10^{-5} \mathrm{~s}^{-1}\right)$ & 11.7 & 1.6 & 46.0 & 7.6
\end{tabular}

1440

SI Table 4: List of photolysis rates measured on the DC-8 and used to constrain the WF 1 and WF2 model runs.

Photolysis Rates Used to Constrain the WF1 and WF2 Model Runs

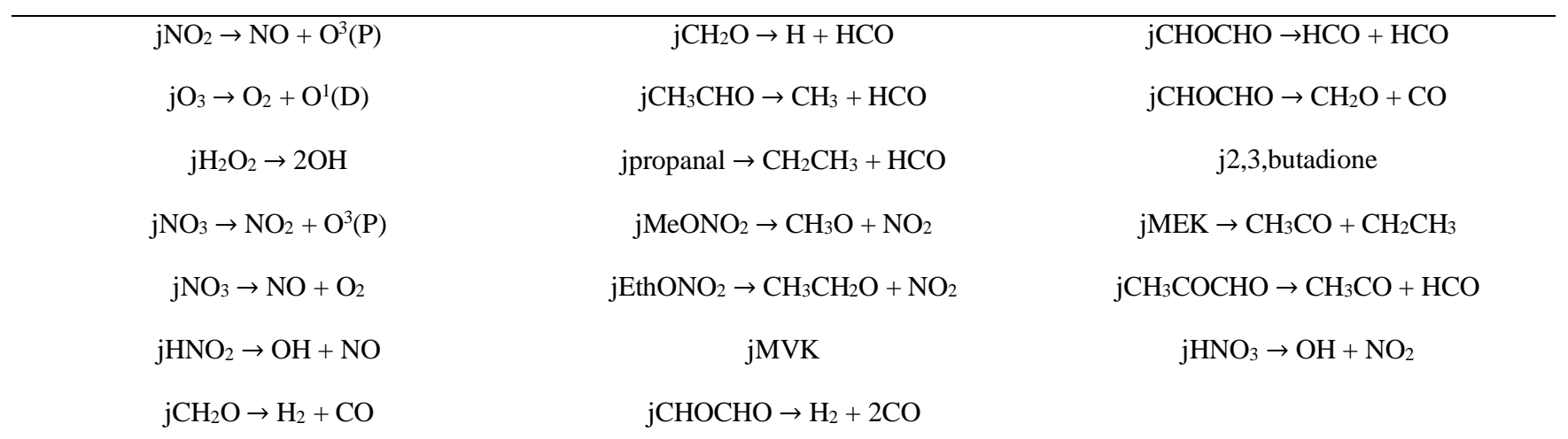

SI Table 5: Table of initial mixing ratios of $\mathrm{CO}, \mathrm{NO}, \mathrm{NO}_{2}, \mathrm{HONO}$, and $\mathrm{O}_{3}$ for all model runs. The Dark model run has the same conditions as the WF2 model run.

\begin{tabular}{|c|c|c|c|c|c|c|c|c|}
\hline & \multicolumn{2}{|c|}{ WF1 } & \multicolumn{2}{|c|}{ WF2 } & \multicolumn{2}{|c|}{ Castle } & \multicolumn{2}{|c|}{ Cow } \\
\hline & ppbv & ppbv ppmv $^{-1}$ & ppbv & ppbv ppmv $^{-1}$ & ppbv & ppbv ppmv $^{-1}$ & ppbv & ppbv ppmv ${ }^{-1}$ \\
\hline $\mathrm{CO}$ & 8259 & - & 8329 & & 1954 & & 2618 & \\
\hline $\mathrm{NO}_{2}$ & 7.0 & 0.8 & 0.0 & 0.0 & 3.6 & 1.8 & 4.2 & 1.6 \\
\hline HONO & 8.0 & 1.0 & 30.0 & 3.6 & 3.0 & 1.5 & 22.5 & 8.6 \\
\hline
\end{tabular}


SI Table 6: Table of observed background $\mathrm{O}_{3}$ during an upwind transect and outside of the plume edges.

\begin{tabular}{l|c|c|c|c} 
& WF1 & WF2 & Castle & Cow \\
\hline Average $\mathrm{O}_{3}$ Upwind (ppbv) & $57.8 \pm 0.4$ & $48.9 \pm 7.5$ & $72 \pm 1$ & - \\
Average $\mathrm{O}_{3}$ Outside of transects (ppbv) & $53 \pm 3$ & $58 \pm 8$ & $82 \pm 2$ & $53 \pm 3$
\end{tabular}

SI Table 7: Comparison of model outputs and observations used (as seen in Figure 2) as the average (Avg) or median (Med) in absolute (ppbv) and percent difference $(\%)$ for all compounds used to iterate the model.

\begin{tabular}{|c|c|c|c|c|c|c|c|c|c|c|c|c|c|c|c|c|}
\hline & \multicolumn{4}{|c|}{ Castle } & \multicolumn{4}{|c|}{ WF1 } & \multicolumn{4}{|c|}{ WF2 } & \multicolumn{4}{|c|}{ Cow } \\
\hline & \multicolumn{2}{|c|}{ (ppbv) } & \multicolumn{2}{|c|}{$(\%)$} & \multicolumn{2}{|c|}{ (ppbv) } & \multicolumn{2}{|c|}{$(\%)$} & \multicolumn{2}{|c|}{ (ppbv) } & \multicolumn{2}{|c|}{$(\%)$} & \multicolumn{2}{|c|}{ (ppbv) } & \multicolumn{2}{|c|}{$(\%)$} \\
\hline & Avg & Med & Avg & Med & Avg & Med & Avg & Med & Avg & Med & Avg & Med & Avg & Med & Avg & Med \\
\hline $\mathrm{CO}$ & 80.5 & 69.9 & 6.8 & 6.5 & 307.4 & 328.2 & 13.1 & 11.7 & 243.1 & 243.1 & 4.1 & 4.2 & 37.8 & 39.7 & 2.8 & 2.8 \\
\hline $\mathrm{NO}_{2}$ & 0.5 & 0.4 & 15.8 & 6.8 & 2.0 & 2.0 & 126.1 & 32.2 & 2.0 & 2.0 & 5.0 & 5.1 & 1.4 & 0.9 & 12.0 & 8.3 \\
\hline NO & 0.3 & 0.2 & 54.9 & 47.6 & 0.4 & 0.1 & 944.6 & 99.8 & 1.0 & 1.0 & 43.7 & 40.5 & 0.1 & 0.1 & 100.0 & 100.0 \\
\hline $\mathrm{O}_{3}$ & 1.6 & 1.7 & 2.0 & 2.1 & 6.1 & 6.3 & 6.5 & 6.2 & 3.4 & 3.4 & 29.7 & 27.2 & 0.4 & 0.3 & 0.8 & 0.8 \\
\hline HONO & 0.3 & 0.4 & 21.6 & 21.8 & 0.9 & 0.8 & 961.4 & 53.3 & 3.4 & 3.4 & 16.9 & 18.8 & 0.5 & 0.4 & 5.5 & 6.6 \\
\hline
\end{tabular}



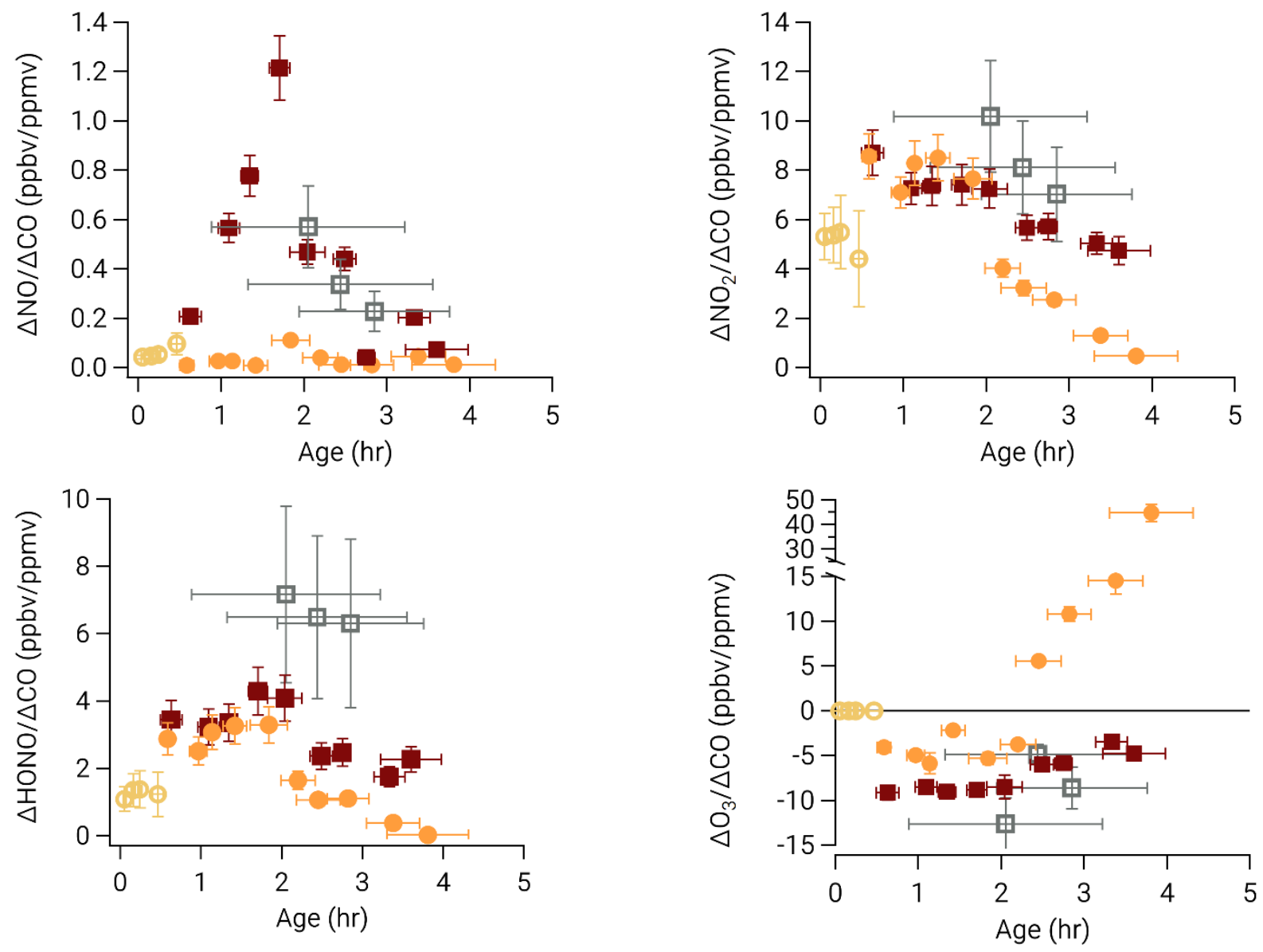

SI Figure 4: Observed normalized excess mixing ratios (NEMRs) of $\mathrm{NO}, \mathrm{NO}_{2}, \mathrm{HONO}$, and $\mathrm{O}_{3}$ for all plumes. 

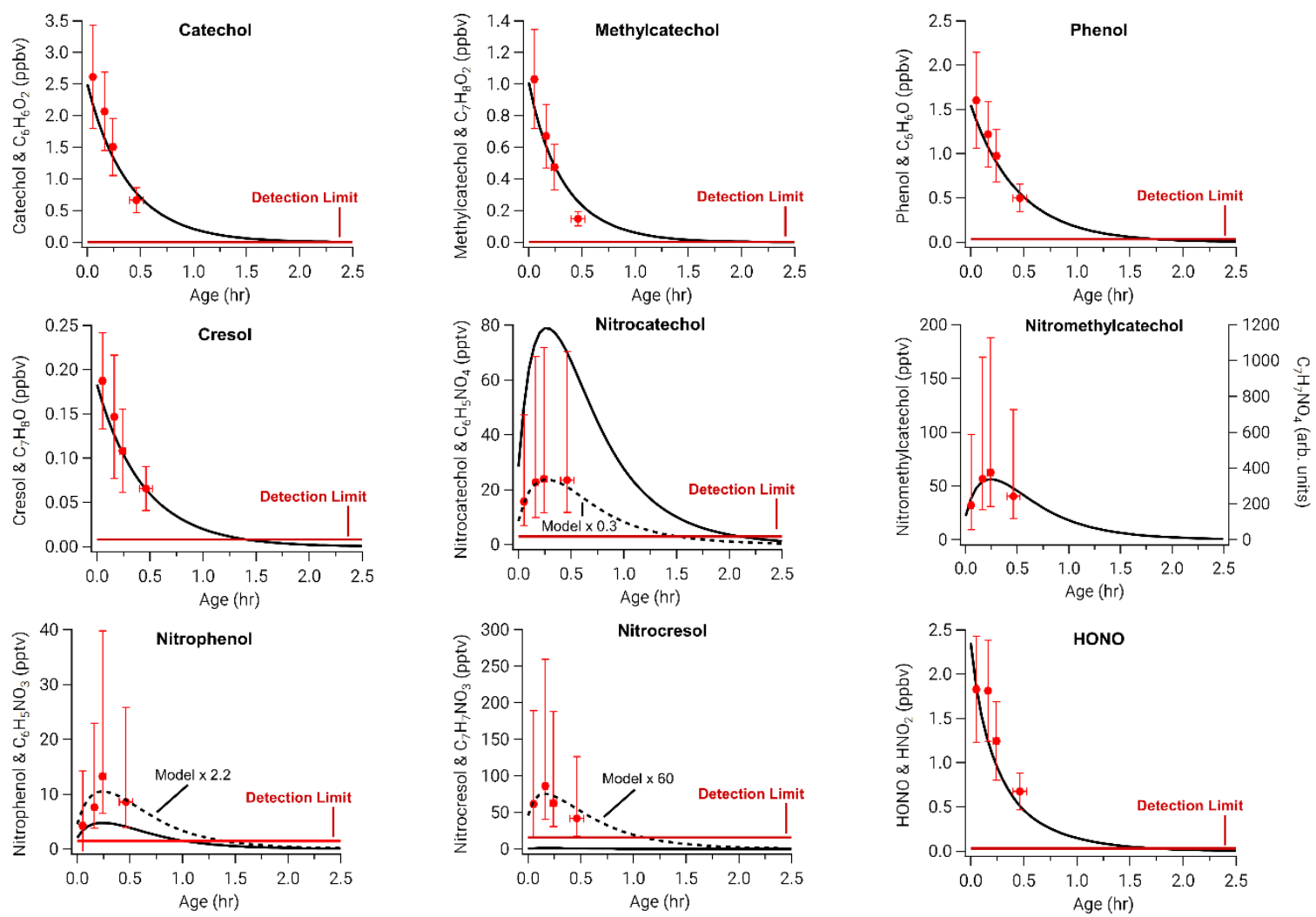

SI Figure 5: Model outputs (black line) and observations (red circles) of the Castle plume where model compounds are indicated by 1460 the name and observations by chemical formula. Observations are made by the University of Washington $\mathbf{I}^{-}$HR ToF CIMS. Detection limits for calibrated compounds are shown as horizontal red lines. For compounds without calibrations we report arbitrary units on the right axis for the purpose of comparing time evolution. 

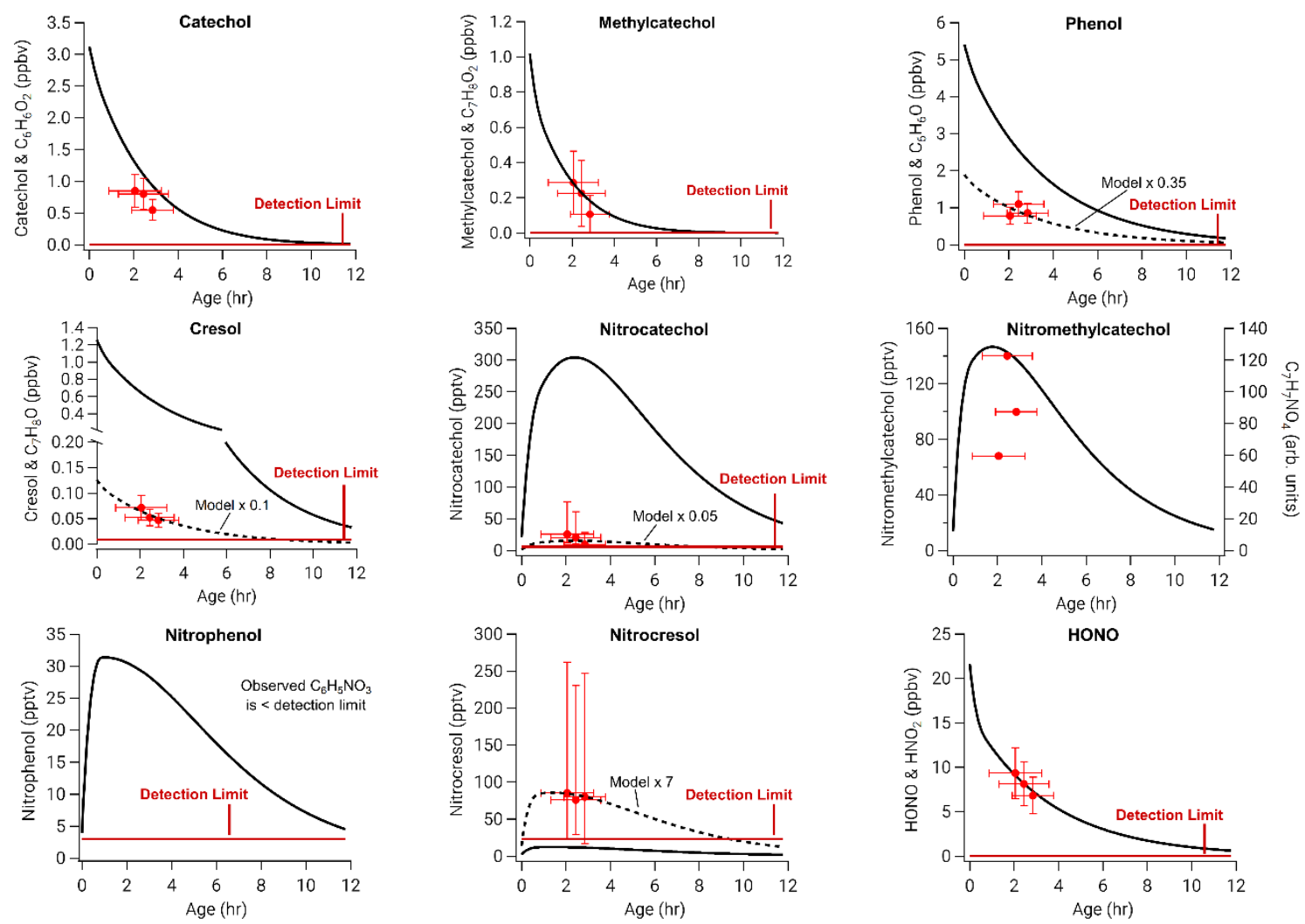

1465 SI Figure 6: Similar to SI Figure 5, but for the Cow plume. 

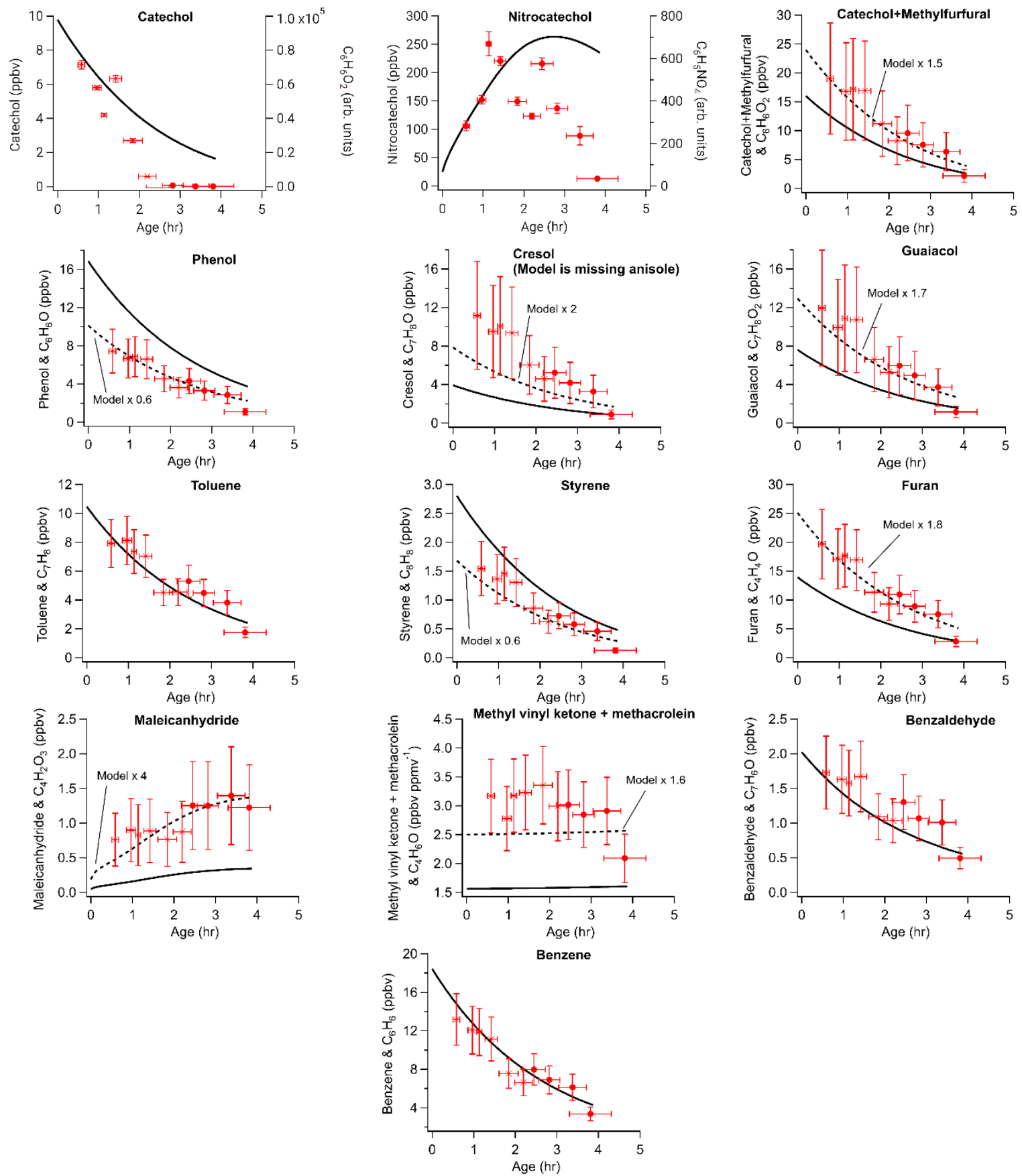

SI Figure 7: Model outputs (black line) and observations (red circles) of the WF1 plume where model compounds are indicated by the name and observations by chemical formula. Observations are made by the NOAA I- CIMS. We report arbitrary units on the right axis for the purpose of comparing time evolution. 

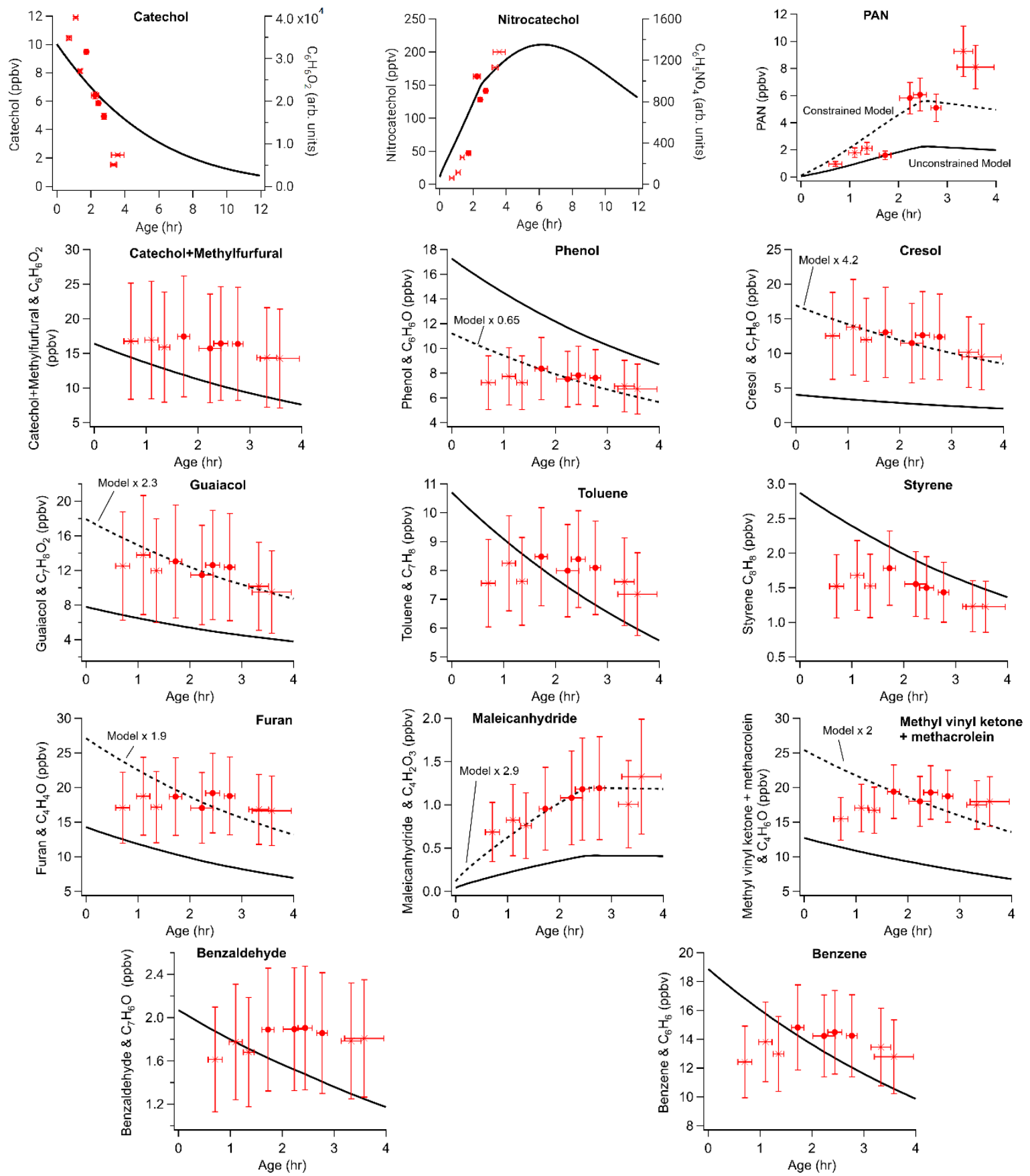

SI Figure 8: Model outputs (black line) and observations (red circles) of the WF2 plume where model compounds are indicated by

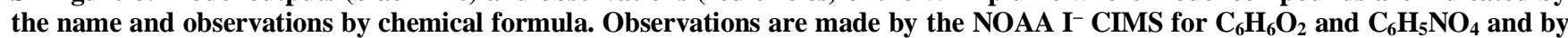
the GT CIMS for PAN. All other compounds were measured by the UIBK PTR ToF MS. For uncalibrated compounds, we report arbitrary units on the right axis for the purpose of comparing time evolution. 

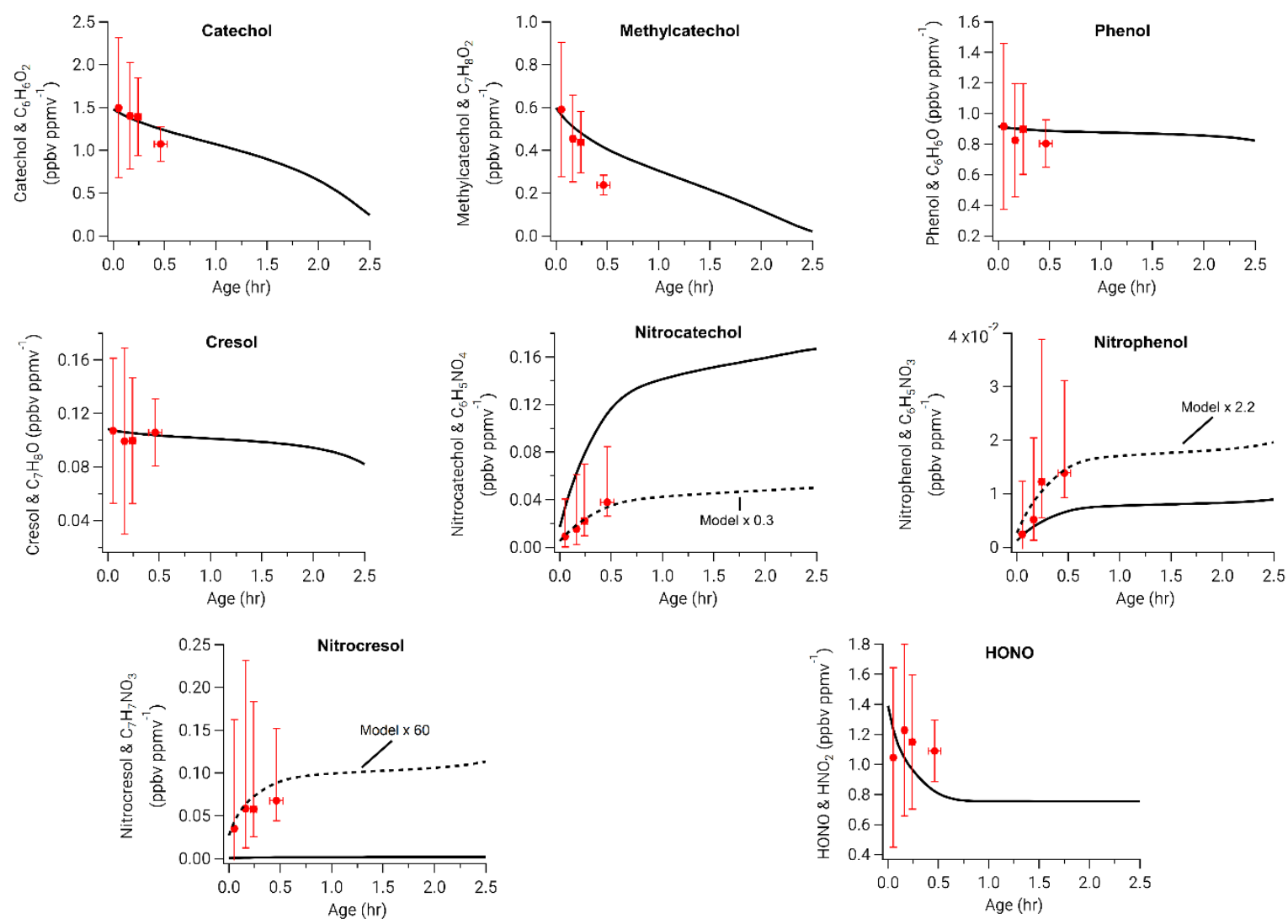

SI Figure 9: Similar to SI Figure 5 (Castle), but in the form of normalized excess mixing ratios (NEMRs) 

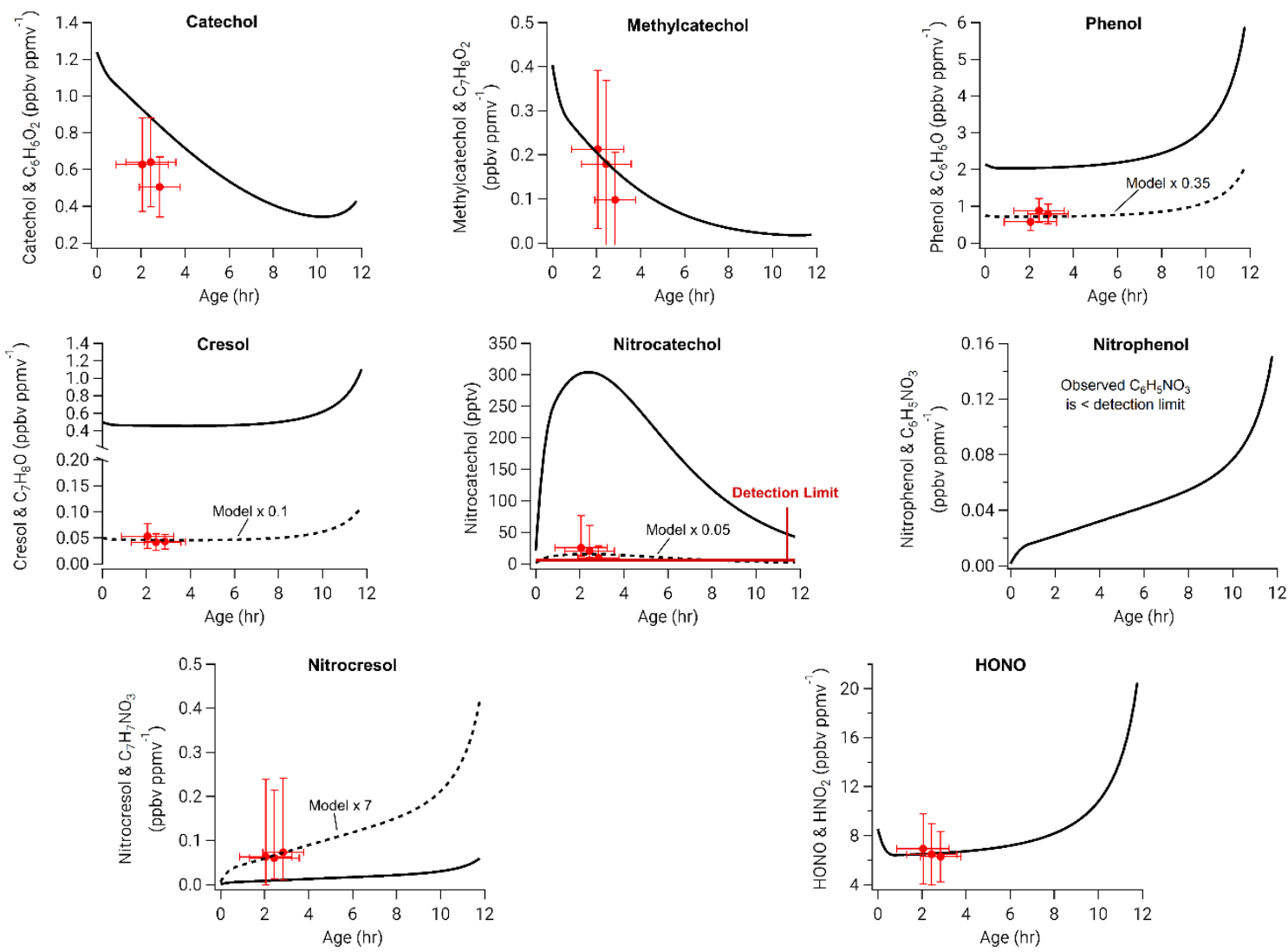

SI Figure 10: Similar to SI Figure 6 (Cow), but in the form of normalized excess mixing ratios (NEMRs). 

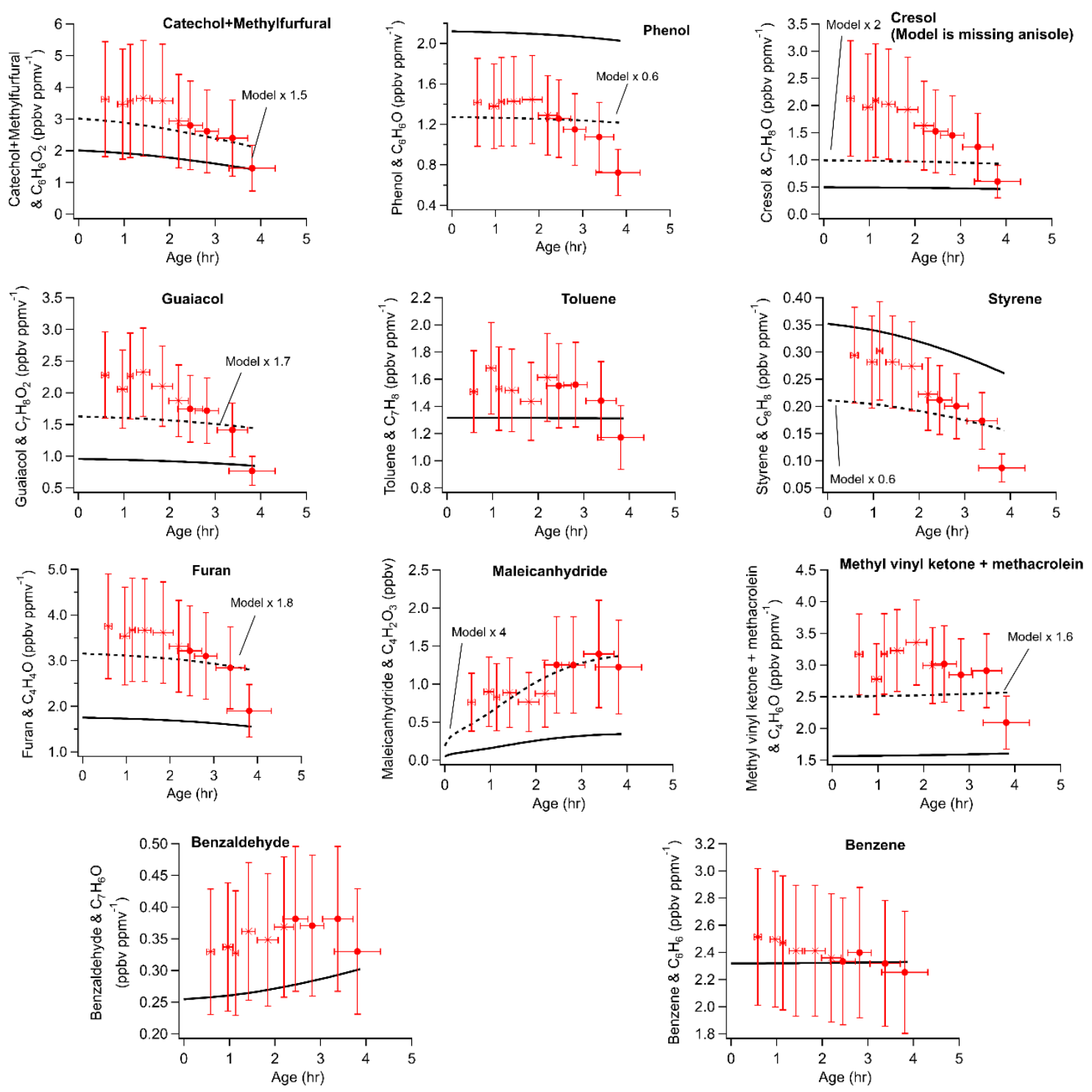

SI Figure 11: Similar to SI Figure 7 (WF1), but in the form of normalixed excess mixing ratios (NEMRs) 

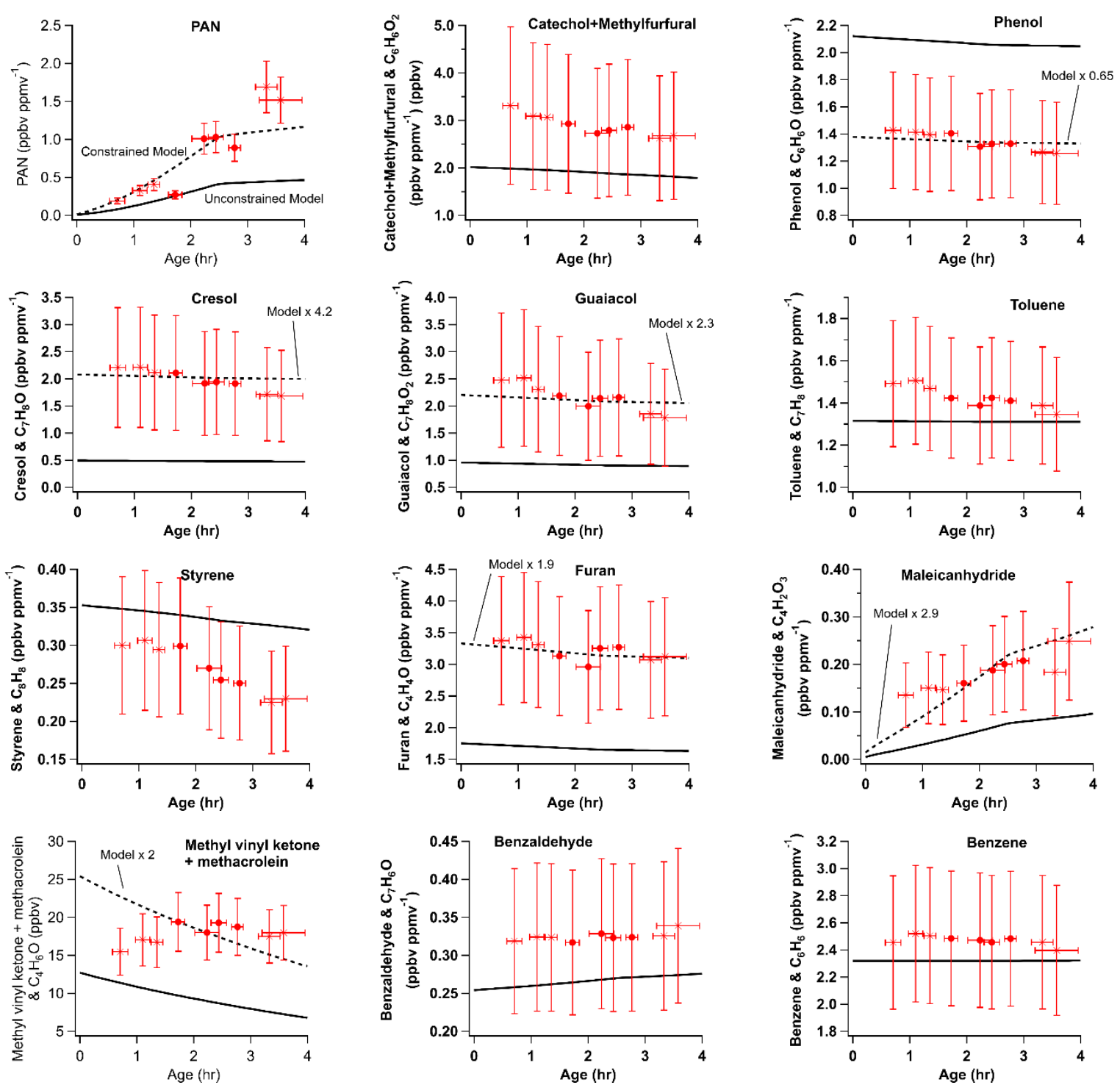

1485 SI Figure 12: Similar to SI Figure 8 (WF2), but in the form of normalixed excess mixing ratios (NEMRs) 

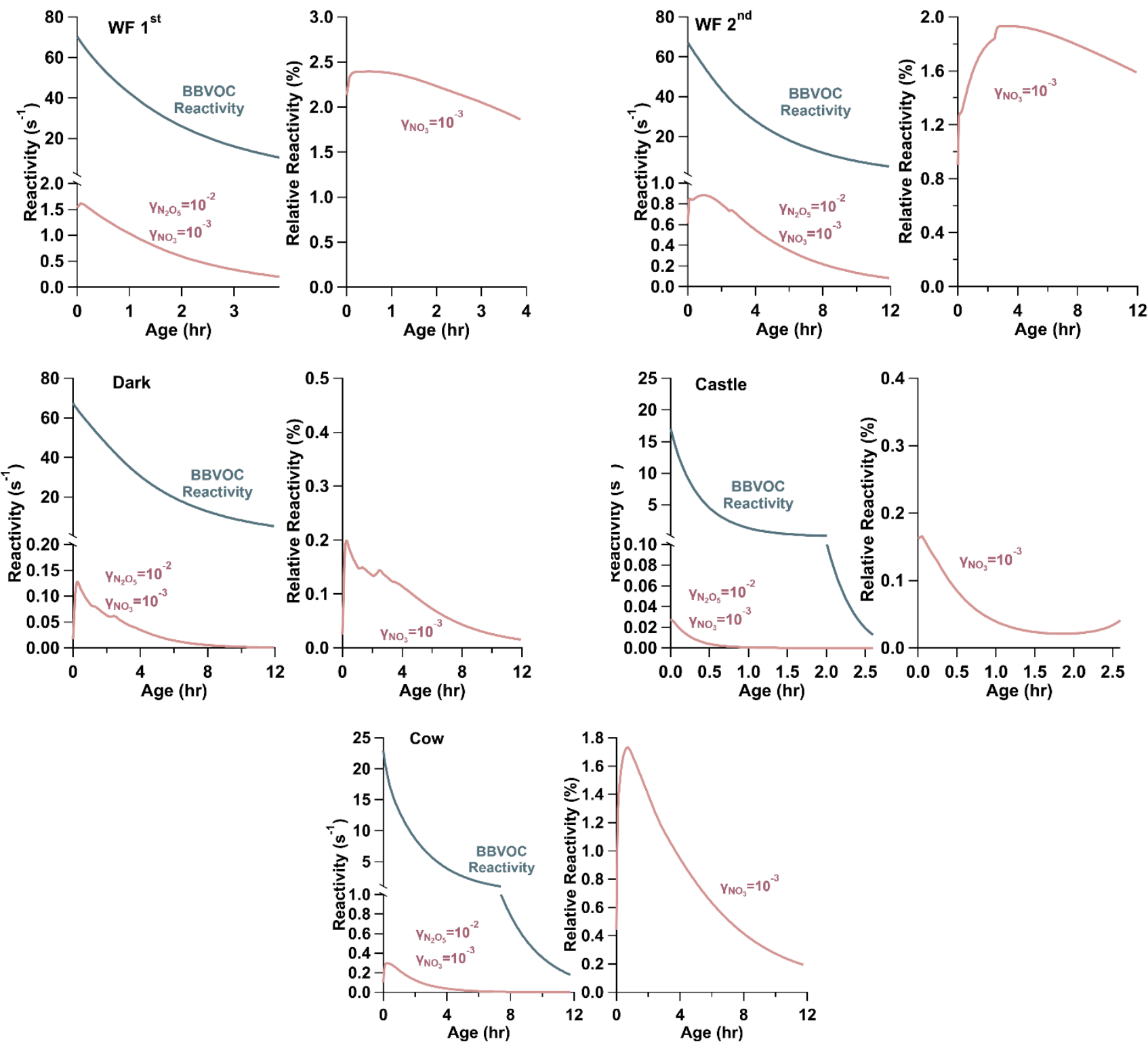

SI Figure 13: Left: total reactivity $\left(\mathrm{s}^{-1}\right)$ of BBVOCs (blue) and $\mathrm{N}_{2} \mathrm{O}_{5} / \mathrm{NO}_{3}$ heterogenous uptake reactivity using a $\gamma_{N_{2} o_{5}}=10^{-2}$ and a $\gamma_{\mathrm{NO}_{3}}=10^{-3}$ (red). Right: Relative reactivity (\%) of $\mathrm{N}_{2} \mathrm{O}_{5} / \mathrm{NO}_{3}$ heterogenous uptake compared to total reactivity (heterogenous uptake $+\mathrm{BBVOCs}$ ) for $\gamma_{\mathrm{NO}_{3}}=1,10^{-1}$, and $10^{-3}$. In all model runs, BBVOCs overwhelmingly control $\mathrm{NO}_{3}$ loss. 

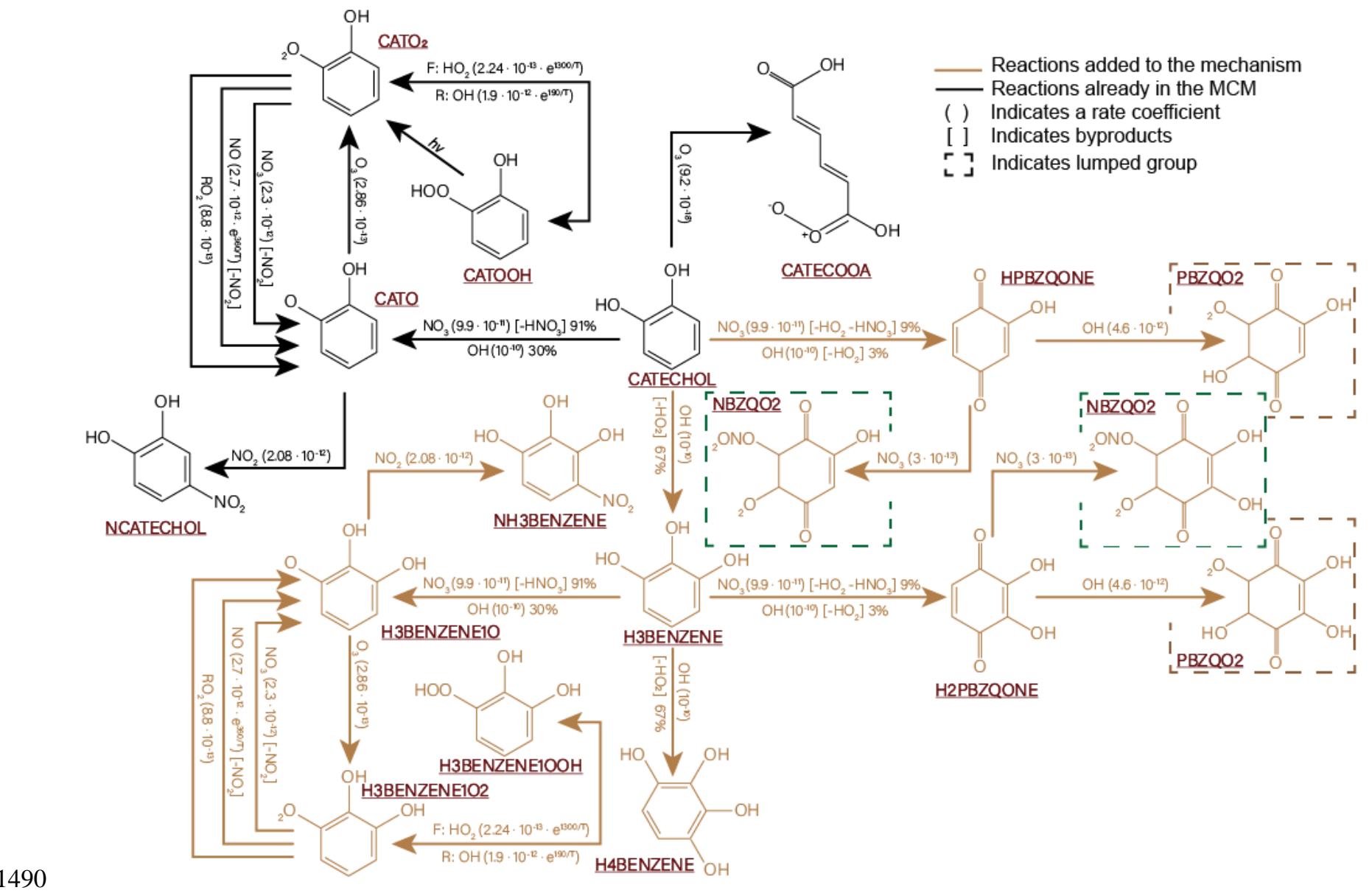

SI Figure 14: Subset of phenolic mechanism expansion showing catechol related reactions only. Reactions that are in the MCM are shown in black, and added reactions are shown in brown. Compounds that we boxed are lumped in the mechanism. Compound names correspond to the provided FACSIMILE provided in the SI. 

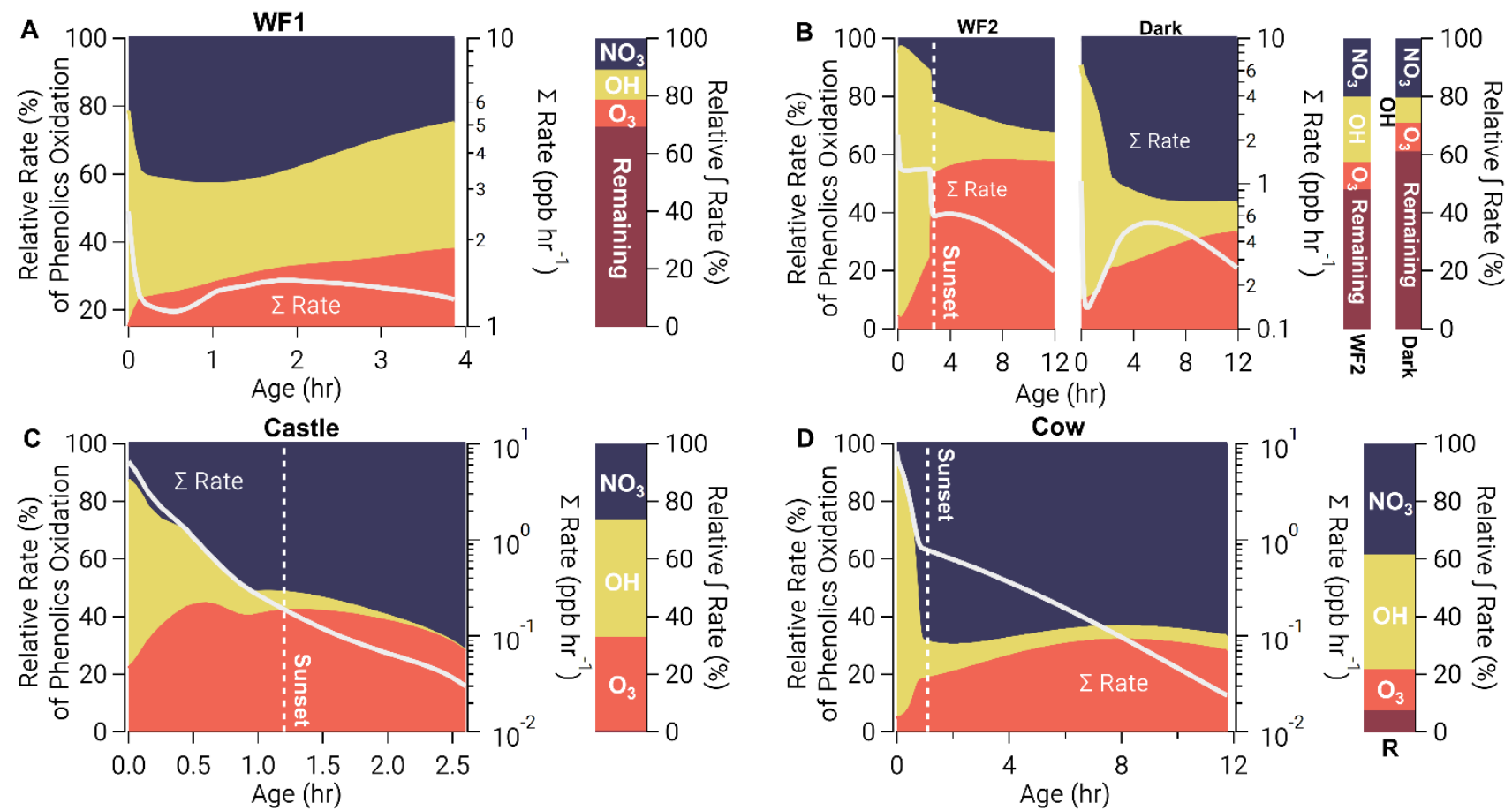

$\mathrm{NO}_{3} \mathrm{OH} \square \mathrm{O}_{3}$

SI Figure 15: Oxidation metrics of all phenolic compounds for WF1 model run (A), WF2 and dark model runs (B), Castle model run (C), and Cow model run (D). Left axis: relative oxidation of phenolics for $\mathrm{NO}_{3}$ (blue), $\mathrm{OH}$ (yellow), and $\mathrm{O}_{3}$ (orange). Right log axis: absolute total oxidation (white line). Bar: Relative integrated rate of oxidation of phenolics for $\mathrm{NO}_{3}$ (blue), $\mathrm{OH}_{(\mathrm{yellow}), \mathrm{O}_{3}}$ (orange) and the remaining phenolics at the model end (red). 

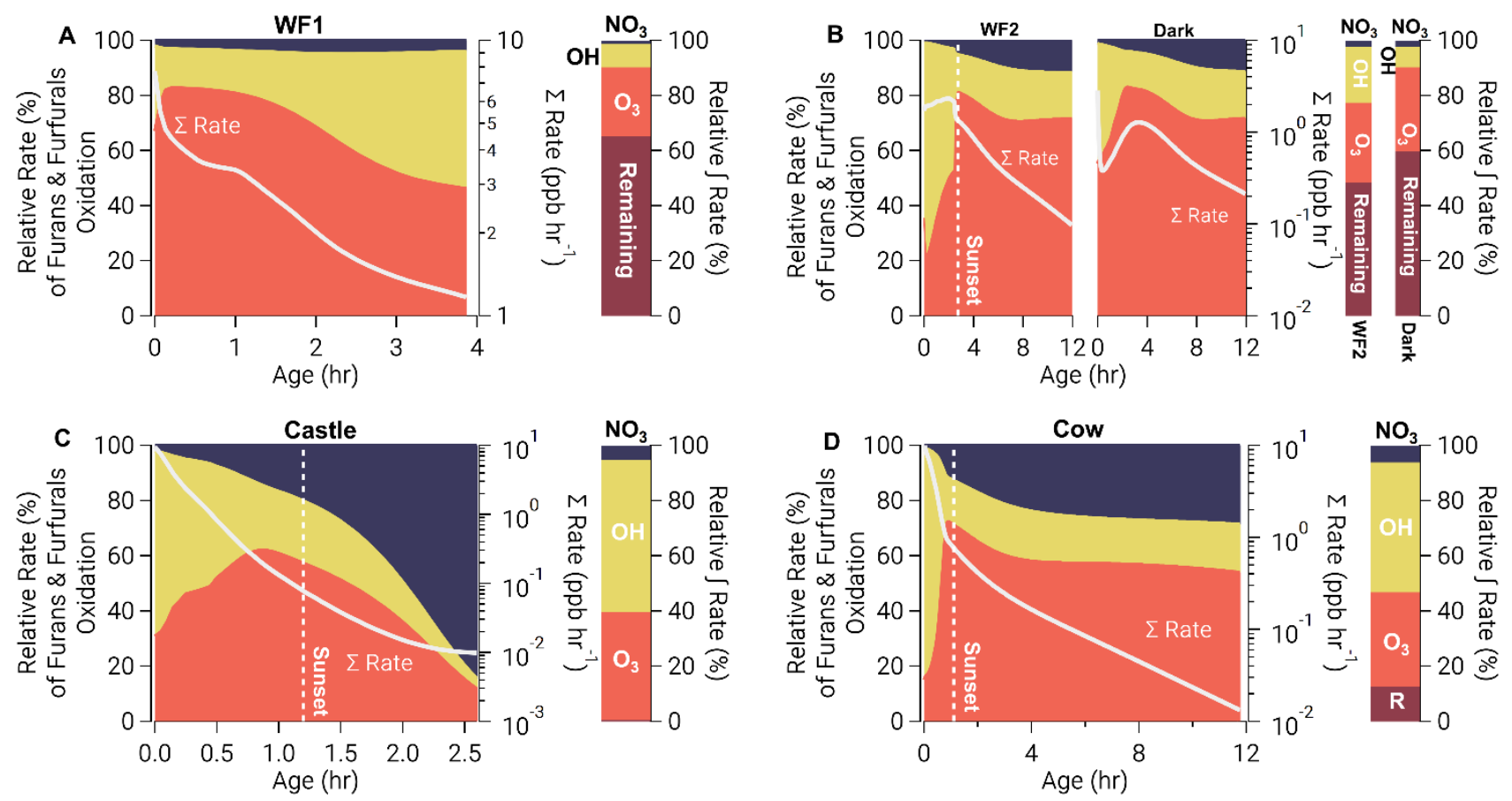

$\mathrm{NO}_{3} \mathrm{OH} \square \mathrm{O}_{3}$

SI Figure 16: Similar to SI Figure 15, but for furans and furfurals. 

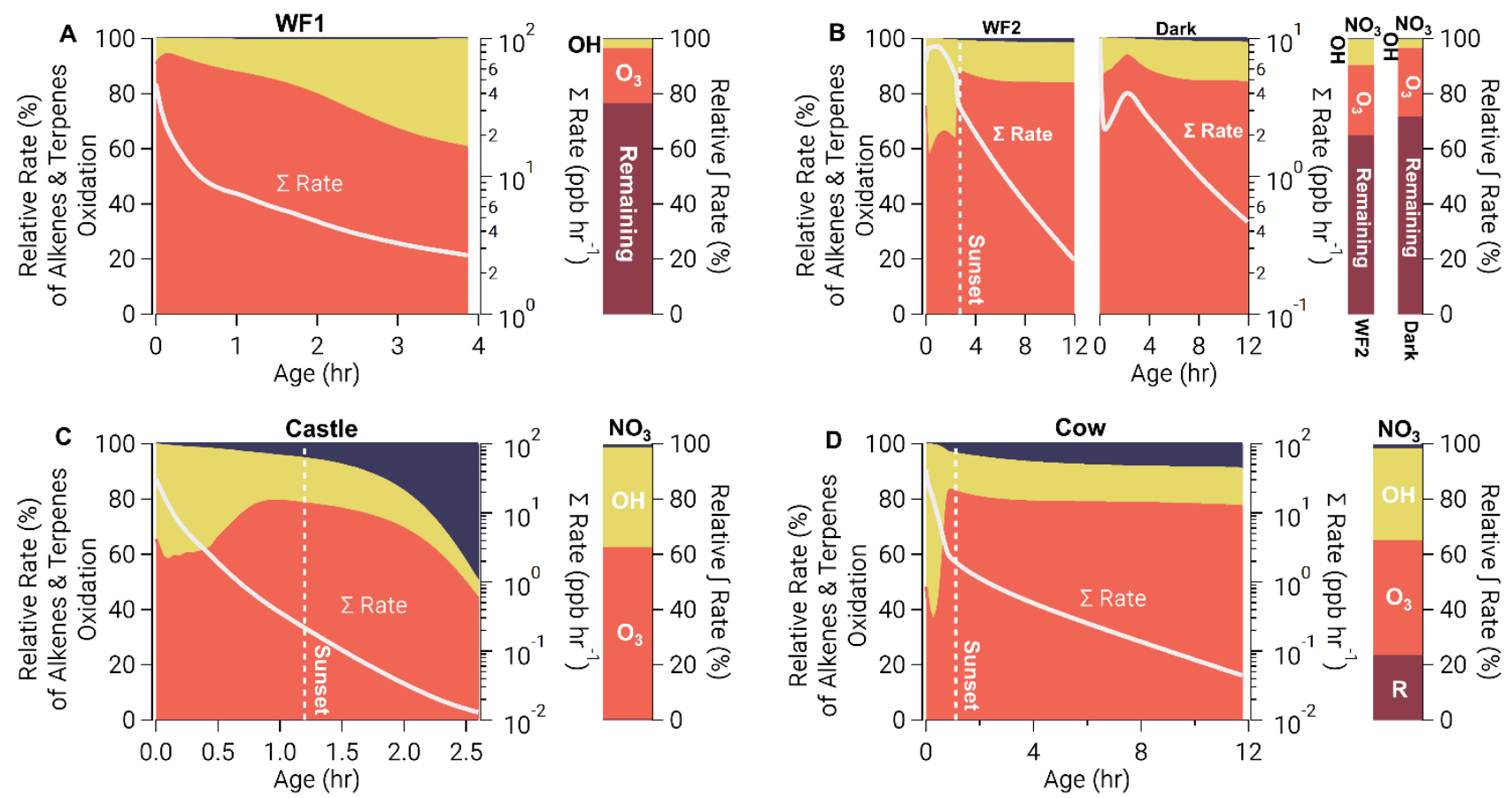

$\mathrm{NO}_{3} \mathrm{OH} \square \mathrm{O}_{3}$

SI Figure 17: Similar to SI Figure 15, but for alkenes. 

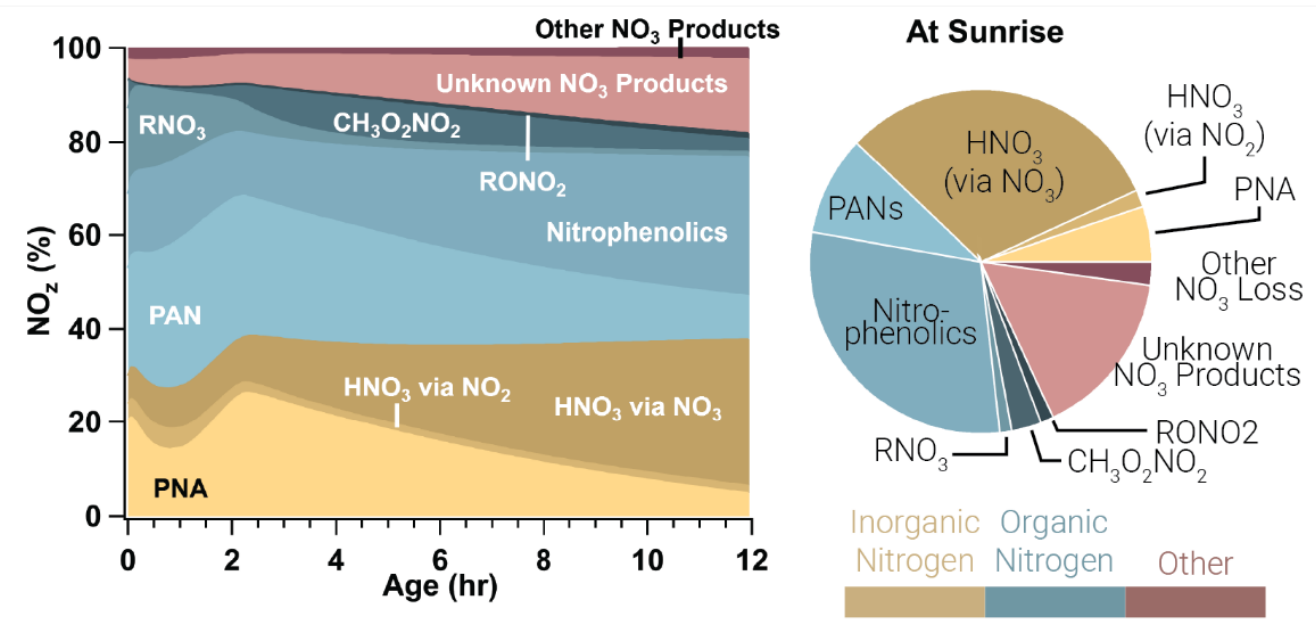

SI Figure 18: Similar to Figure 10 in the main text, but for the Dark model run. 